\title{
On a Five-Dimensional Nonautonomous Schistosomiasis Model with Latent Period
}

\author{
Shujing Gao, ${ }^{1}$ Yanfei Dai, ${ }^{2}$ and Dehui Xie ${ }^{1}$ \\ ${ }^{1}$ Key Laboratory of Jiangxi Province for Numerical Simulation and Emulation Techniques, Gannan Normal University, \\ Ganzhou 341000, China \\ ${ }^{2}$ School of Mathematics, Sun Yat-sen University, Guangzhou 510275, China
}

Correspondence should be addressed to Shujing Gao; gaosjmath@126.com

Received 2 June 2016; Accepted 4 August 2016

Academic Editor: Darko Mitrovic

Copyright (C) 2016 Shujing Gao et al. This is an open access article distributed under the Creative Commons Attribution License, which permits unrestricted use, distribution, and reproduction in any medium, provided the original work is properly cited.

\begin{abstract}
A five-dimensional nonautonomous schistosomiasis model which include latent period is proposed and studied. By constructing several auxiliary functions and using some skills, we obtain some sufficient conditions for the extinction and permanence (uniform persistence) of infectious population of the model. New threshold values of integral form are obtained. For the corresponding autonomous schistosomiasis model, our results are consistent with the past results. For the periodic and almost periodic cases, some corollaries for the extinction and permanence of the disease are established. In order to illustrate our theoretical analysis, some numerical simulations are presented.
\end{abstract}

\section{Introduction}

Schistosomiasis is a chronic, parasitic disease caused by blood flukes (trematode worms) of the genus Schistosoma. More than 207 million people, $85 \%$ of them living in Africa, are infected worldwide, with an estimated 700 million people at risk in 74 endemic countries. Schistosomiasis is prevalent in tropical and subtropical areas, especially in poor communities without access to safe drinking water and adequate sanitation [1]. In the developing countries, schistosomiasis is frequently a serious health problem [2].

The first mathematical models for schistosomiasis were those developed by Macdonald in [3] and Hairston in [4]. Since then, a number of mathematical models have been proposed. Barbour [5] studied the prevalence of infection in the definitive host population and the snails, Chiyaka and Garira [6] were concerned with the combined effect of the inclusion the dynamics of the miracidia and cercariae populations and a density dependent infection term, Bhunu et al. [7] developed a mathematical model of the transmission dynamics of HIV/AIDS in the presence of schistosomiasis in the human-snail hosts, Yang and Xiao [8] presented a dynamic model of Schistosoma japonicum transmission that incorporated effects of the prepatent periods of the different stages of Schistosoma into Barbour's model, Qi and Cui [9] established a diffusive system of partial differential equations considering the diffusion of human and snail hosts, and Qi et al. [10] developed a schistosomiasis model of the prevalence of infection in the Rattus norvegicus and Oncomelania snail, in which the authors considered a schistosomiasis model on the Rattus norvegicus in Qianzhou and Zimuzhou, two islets in the center of Yangtze River near Nanjing, China. There have been no human residents or other livestock on the islets; thus the mammalian Rattus norvegicus is the only definitive host while snail serves as the unique intermediate host; the authors set up the compartmental model as follows:

$$
\begin{aligned}
& \frac{d x_{s}}{d t}=A_{x}-\mu_{x} x_{s}-\beta_{x} x_{s} y_{i}, \\
& \frac{d x_{i}}{d t}=\beta_{x} x_{s} y_{i}-\mu_{x} x_{i}-\alpha_{x} x_{i}, \\
& \frac{d y_{s}}{d t}=A_{y}-\mu_{y} y_{s}-\beta_{y} x_{i} y_{s},
\end{aligned}
$$




$$
\begin{aligned}
& \frac{d y_{e}}{d t}=\beta_{y} x_{i} y_{s}-\left(\mu_{y}+\theta\right) y_{e}, \\
& \frac{d y_{i}}{d t}=\theta y_{e}-\mu_{y} y_{i}-\alpha_{y} y_{i} .
\end{aligned}
$$

Here, $x_{s}, x_{i}$ represent the number of susceptible and infected Rattus norvegicus, respectively. $y_{s}, y_{e}, y_{i}$ are the number of susceptible, infected and preshedding, and infected and shedding Oncomelania snail, respectively. The other parameters interpretation of model (1) is shown in Parameters Section. All the parameters in the model are assumed to be nonnegative constants.

According to the concept of the next generation of matrix [11], the authors obtain the basic reproduction number [12] of system (1) as follows:

$$
R_{0}=\sqrt[3]{\frac{A_{x} A_{y} \theta \beta_{x} \beta_{y}}{\mu_{x} \mu_{y}\left(\mu_{x}+\alpha_{x}\right)\left(\mu_{y}+\alpha_{y}\right)\left(\mu_{y}+\theta\right)}} .
$$

And they prove that the infection will go extinct if $R_{0} \leq 1$ and schistosomiasis will be prevalent if $R_{0}>1$.

Nonautonomous phenomena, however, are dominating in real epidemic systems. Many diseases show seasonal behavior and taking into account the seasonal influence in epidemic models is very necessary. The nonautonomous phenomenon come from various sources, such as the variation of transmission rate and fluctuations in death and birth rates. Particularly, these coefficients of models usually vary with time when long-time dynamical behaviors are studied for an epidemic system. More and more realistic and interesting nonautonomous models can be found in papers [13-24] and the references cited therein.

Motivated by the ideas of the above aspects, system (1) is modified to take into account factors mentioned above where all coefficients vary with time to give a more appropriate result and better understanding of the prevalence of schistosomiasis. Thus, we refer to the following nonautonomous system for the study of schistosomiasis:

$$
\begin{aligned}
\frac{d x_{s}(t)}{d t}= & A_{x}(t)-\mu_{x}(t) x_{s}(t)-\beta_{x}(t) x_{s}(t) y_{i}(t), \\
\frac{d x_{i}(t)}{d t}= & \beta_{x}(t) x_{s}(t) y_{i}(t)-\mu_{x}(t) x_{i}(t) \\
& -\alpha_{x}(t) x_{i}(t), \\
\frac{d y_{s}(t)}{d t}= & A_{y}(t)-\mu_{y}(t) y_{s}(t)-\beta_{y}(t) x_{i}(t) y_{s}(t), \\
\frac{d y_{e}(t)}{d t}= & \beta_{y}(t) x_{i}(t) y_{s}(t)-\left(\mu_{y}(t)+\theta(t)\right) y_{e}(t), \\
\frac{d y_{i}(t)}{d t}= & \theta(t) y_{e}(t)-\mu_{y}(t) y_{i}(t)-\alpha_{y}(t) y_{i}(t)
\end{aligned}
$$

with initial value

$$
\begin{aligned}
& x_{s}(0)>0, \\
& x_{i}(0)>0, \\
& y_{s}(0)>0, \\
& y_{e}(0) \geq 0, \\
& y_{i}(0)>0 .
\end{aligned}
$$

In this paper, our purpose is to obtain the weaker conditions for the permanence and extinction of schistosomiasis. We will establish the sufficient conditions for the permanence and extinction of the disease and give some new threshold values of the integral form. When system (3) reduces to periodic or almost periodic case, the basic reproduction number is obtained (see Corollaries 18 and 19 given in Section 5). Our results show that the threshold value acts as a sharp threshold for the permanence and extinction of the disease.

This paper is organized as follows. In Section 2 we present preliminary setting and propositions which are used to analyze the long-time behavior of system (3) in the following sections. In Sections 3 and 4, we prove our main theorems on the extinction and permanence of infectious population of system (3). In Section 5, we derive some corollaries for the extinction and permanence of infectious population of system (3) for some special cases. In Section 6, we provide numerical examples to illustrate the validity of our analytical results.

\section{Notations and Preliminaries}

At first, we put the following assumptions for system (3).

(H1) Functions $A_{x}(t), \mu_{x}(t), \beta_{x}(t), \alpha_{x}(t), A_{y}(t), \mu_{y}(t)$, $\beta_{y}(t), \alpha_{y}(t)$, and $\theta(t)$ are positive, bounded, and continuous on $[0,+\infty)$.

(H2) There exist constants $\omega_{i}>0(i=1,2,3,4,5,6)$ such that

$$
\begin{aligned}
& \liminf _{t \rightarrow+\infty} \int_{t}^{t+\omega_{1}} A_{x}(s) d s>0, \\
& \liminf _{t \rightarrow+\infty} \int_{t}^{t+\omega_{2}} A_{y}(s) d s>0, \\
& \liminf _{t \rightarrow+\infty} \int_{t}^{t+\omega_{3}} \mu_{x}(s) d s>0, \\
& \liminf _{t \rightarrow+\infty} \int_{t}^{t+\omega_{4}} \mu_{y}(s) d s>0, \\
& \liminf _{t \rightarrow+\infty} \int_{t}^{t+\omega_{5}} \beta_{x}(s) d s>0, \\
& \liminf _{t \rightarrow+\infty} \int_{t}^{t+\omega_{6}} \beta_{y}(s) d s>0 .
\end{aligned}
$$


For any continuous periodic function $f$ with period $\omega>$ 0 , we denote by $\bar{f}$ the average value of $f(t)$ where $\bar{f}=$ $(1 / \omega) \int_{0}^{\omega} f(t) d t$.

For any continuous almost periodic function $f$, we denote by $m(f)$ the average value of $f(t)$ where $m(f)=$ $\lim _{t \rightarrow+\infty}(1 / t) \int_{0}^{t} f(t) d t$.

Remark 1. When system (3) degenerates into $\omega$-periodic system, that is to say, all the coefficients of system (3) are all nonnegative and nonzero, continuous periodic functions with period $\omega>0$, then $(\mathrm{H} 2)$ is equivalent to the following cases:

$$
\begin{aligned}
& \bar{A}_{x}>0, \\
& \bar{A}_{y}>0, \\
& \bar{\mu}_{x}>0, \\
& \bar{\mu}_{y}>0, \\
& \bar{\beta}_{x}>0, \\
& \bar{\beta}_{y}>0 .
\end{aligned}
$$

Remark 2. When system (3) degenerates into almost periodic system, that is to say, all the coefficients in it are all nonnegative and nonzero, continuous almost periodic functions, then (H2) is equivalent to the following cases:

$$
\begin{gathered}
m\left(A_{x}\right)>0, \\
m\left(A_{y}\right)>0, \\
m\left(\mu_{x}\right)>0, \\
m\left(\mu_{y}\right)>0, \\
m\left(\beta_{x}\right)>0, \\
m\left(\beta_{y}\right)>0 .
\end{gathered}
$$

In what follows, we denote $N_{x}(t):=x_{s}(t)+x_{i}(t), N_{y}(t):=$ $y_{s}(t)+y_{e}(t)+y_{i}(t)$. We also define that $\underline{N}_{x}(t), \bar{N}_{x}(t), \underline{N}_{y}(t)$, $\bar{N}_{y}(t), \underline{x}_{s}(t), \underline{y}_{s}(t)$ are the solutions of the following equations with initial value $\underline{x}_{s}(0)=x_{s}(0)>0, \underline{y}_{s}(0)=y_{s}(0)>0$, $\underline{N}_{x}(0)=\bar{N}_{x}(0)=N_{x}(0)=x_{s}(0)+x_{i}(0), \underline{N}_{y}(0)=\bar{N}_{y}(0)=$ $N_{y}(0)=y_{s}(0)+y_{e}(0)+y_{i}(0)$, respectively:

$$
\begin{aligned}
& \frac{d N_{x}(t)}{d t}=A_{x}(t)-\left(\mu_{x}(t)+\alpha_{x}(t)\right) N_{x}(t), \\
& \frac{d N_{x}(t)}{d t}=A_{x}(t)-\mu_{x}(t) N_{x}(t), \\
& \frac{d N_{y}(t)}{d t}=A_{y}(t)-\left(\mu_{y}(t)+\alpha_{y}(t)\right) N_{y}(t), \\
& \frac{d N_{y}(t)}{d t}=A_{y}(t)-\mu_{y}(t) N_{y}(t),
\end{aligned}
$$

$$
\begin{aligned}
& \frac{d x_{s}(t)}{d t}=A_{x}(t)-\left(\mu_{x}(t)+\beta_{x}(t) \bar{N}_{y}(t)\right) x_{s}(t), \\
& \frac{d y_{s}(t)}{d t}=A_{y}(t)-\left(\mu_{y}(t)+\beta_{y}(t) \bar{N}_{x}(t)\right) y_{s}(t) .
\end{aligned}
$$

Obviously, from system (3) and the comparison theorem, we have

$$
\begin{gathered}
\underline{N}_{x}(t) \leq N_{x}(t) \leq \bar{N}_{x}(t), \\
\underline{N}_{y}(t) \leq N_{y}(t) \leq \bar{N}_{y}(t), \\
\underline{x}_{s}(t) \leq x_{s}(t) \leq \bar{N}_{x}(t), \\
\underline{y}_{s}(t) \leq y_{s}(t) \leq \bar{N}_{y}(t) .
\end{gathered}
$$

Furthermore, we have the following result.

Lemma 3. Suppose that assumptions (H1) and (H2) hold. Then the following results hold.

(a) There exist constants $m_{1}>0$ and $M_{1}>0$, which are independent of the choice of initial value $N_{x}(0)>0$, such that

$$
\begin{aligned}
0 & <m_{1}<\liminf _{t \rightarrow+\infty} \underline{N}_{x}(t) \leq \liminf _{t \rightarrow+\infty} N_{x}(t) \\
& \leq \limsup _{t \rightarrow+\infty} N_{x}(t) \leq \limsup _{t \rightarrow+\infty} \bar{N}_{x}(t)<M_{1}<+\infty .
\end{aligned}
$$

(b) There exist constants $m_{2}>0$ and $M_{2}>0$, which are independent of the choice of initial value $N_{y}(0)>0$, such that

$$
\begin{aligned}
0 & <m_{2}<\liminf _{t \rightarrow+\infty} \underline{\underline{N}}_{y}(t) \leq \liminf _{t \rightarrow+\infty} N_{y}(t) \\
& \leq \limsup _{t \rightarrow+\infty} N_{y}(t) \leq \limsup _{t \rightarrow+\infty} \bar{N}_{y}(t)<M_{2}<+\infty .
\end{aligned}
$$

(c) Each fixed solution $\underline{N}_{x}(t)$ of (8), $\bar{N}_{x}(t)$ of (9), $\underline{N}_{y}(t)$ of (10), and $\bar{N}_{y}(t)$ of (11) is bounded and globally uniformly attractive on $R_{+}$.

(d) When (8) is w-periodic, then (8) has a unique nonnegative $\omega$-periodic solution $\underline{N}_{x}(t)$ which is globally uniformly attractive. And (9)-(11) have similar results.

(e) When (8) is almost periodic, then (8) has a unique nonnegative almost periodic solution $N_{x}(t)$ which is globally uniformly attractive. And (9)-(11) have similar results.

(f) If $\mu_{x}(t)>0$ for all $t \geq 0$ and $0<\liminf _{t \rightarrow \infty}\left(A_{x}(t) /\right.$ $\left.\left(\mu_{x}(t)+\alpha_{x}(t)\right)\right) \leq \lim \sup _{t \rightarrow \infty}\left(A_{x}(t) /\left(\mu_{x}(t)+\alpha_{x}(t)\right)\right)<$ $\infty$, then for any solution $\underline{N}_{x}(t)$ of $(8)$, we have

$$
\begin{aligned}
\left(\frac{A_{x}}{\mu_{x}+\alpha_{x}}\right)^{m} & \leq \liminf _{t \rightarrow+\infty} \underline{N}_{x}(t) \leq \limsup _{t \rightarrow+\infty} \underline{N}_{x}(t) \\
& \leq\left(\frac{A_{x}}{\mu_{x}+\alpha_{x}}\right)^{M},
\end{aligned}
$$


where

$$
\begin{aligned}
& \left(\frac{A_{x}}{\mu_{x}+\alpha_{x}}\right)^{m}=\liminf _{t \rightarrow+\infty} \frac{A_{x}(t)}{\mu_{x}(t)+\alpha_{x}(t)}, \\
& \left(\frac{A_{x}}{\mu_{x}+\alpha_{x}}\right)^{M}=\limsup _{t \rightarrow+\infty} \frac{A_{x}(t)}{\mu_{x}(t)+\alpha_{x}(t)} .
\end{aligned}
$$

(g) If $\mu_{x}(t)>0$ for all $t \geq 0$ and $0<\liminf _{t \rightarrow \infty}\left(A_{x}(t) /\right.$ $\left.\mu_{x}(t)\right) \leq \lim \sup _{t \rightarrow \infty}\left(A_{x}(t) / \mu_{x}(t)\right)<\infty$, then, for any solution $\bar{N}_{x}(t)$ of $(9)$, we have

$$
\begin{aligned}
\left(\frac{A_{x}}{\mu_{x}}\right)^{m} & \leq \liminf _{t \rightarrow+\infty} \bar{N}_{x}(t) \leq \limsup _{t \rightarrow+\infty} \bar{N}_{x}(t) \\
& \leq\left(\frac{A_{x}}{\mu_{x}}\right)^{M},
\end{aligned}
$$

where

$$
\begin{aligned}
& \left(\frac{A_{x}}{\mu_{x}}\right)^{m}=\liminf _{t \rightarrow+\infty} \frac{A_{x}(t)}{\mu_{x}(t)} \\
& \left(\frac{A_{x}}{\mu_{x}}\right)^{M}=\limsup _{t \rightarrow+\infty} \frac{A_{x}(t)}{\mu_{x}(t)} .
\end{aligned}
$$

(h) If $\mu_{y}(t)>0$ for all $t \geq 0$ and $0<\liminf _{t \rightarrow \infty}\left(A_{y}(t) /\right.$ $\left.\left(\mu_{y}(t)+\alpha_{y}(t)\right)\right) \leq \lim \sup _{t \rightarrow \infty}\left(A_{y}(t) /\left(\mu_{y}(t)+\alpha_{y}(t)\right)\right)<$ $\infty$, then, for any solution $\underline{N}_{y}(t)$ of $(10)$, we have

$$
\begin{aligned}
\left(\frac{A_{y}}{\mu_{y}+\alpha_{y}}\right)^{m} & \leq \liminf _{t \rightarrow+\infty} \underline{N}_{y}(t) \leq \limsup _{t \rightarrow+\infty} \underline{N}_{y}(t) \\
& \leq\left(\frac{A_{y}}{\mu_{y}+\alpha_{y}}\right)^{M},
\end{aligned}
$$

where

$$
\begin{aligned}
& \left(\frac{A_{y}}{\mu_{y}+\alpha_{y}}\right)^{m}=\liminf _{t \rightarrow+\infty} \frac{A_{y}(t)}{\mu_{y}(t)+\alpha_{y}(t)}, \\
& \left(\frac{A_{y}}{\mu_{y}+\alpha_{y}}\right)^{M}=\limsup _{t \rightarrow+\infty} \frac{A_{y}(t)}{\mu_{y}(t)+\alpha_{y}(t)} .
\end{aligned}
$$

(i) If $\mu_{y}(t)>0$ for all $t \geq 0$ and $0<\liminf _{t \rightarrow \infty}\left(A_{y}(t) /\right.$ $\left.\mu_{y}(t)\right) \leq \limsup _{t \rightarrow \infty}\left(A_{y}(t) / \mu_{y}(t)\right)<\infty$, then, for any solution $\bar{N}_{y}(t)$ of (11), we have

$$
\begin{aligned}
\left(\frac{A_{y}}{\mu_{y}}\right)^{m} & \leq \liminf _{t \rightarrow+\infty} \bar{N}_{y}(t) \leq \limsup _{t \rightarrow+\infty} \bar{N}_{y}(t) \\
& \leq\left(\frac{A_{y}}{\mu_{y}}\right)^{M},
\end{aligned}
$$

where

$$
\begin{aligned}
& \left(\frac{A_{y}}{\mu_{y}}\right)^{m}=\liminf _{t \rightarrow+\infty} \frac{A_{y}(t)}{\mu_{y}(t)}, \\
& \left(\frac{A_{y}}{\mu_{y}}\right)^{M}=\limsup _{t \rightarrow+\infty} \frac{A_{y}(t)}{\mu_{y}(t)} .
\end{aligned}
$$

Lemma 4. Suppose that assumptions (H1) and (H2) hold. Then the solution $\left(x_{s}(t), x_{i}(t), y_{s}(t), y_{e}(t), y_{i}(t)\right)$ of system (3) with initial value (3) exists and is uniformly bounded. Furthermore, for any $t>0$, we have

$$
\begin{aligned}
& x_{s}(t)>0, \\
& x_{i}(t)>0, \\
& y_{s}(t)>0, \\
& y_{e}(t) \geq 0, \\
& y_{i}(t)>0 .
\end{aligned}
$$

For any $p>0, q>0$, and $t>0$ we define

$$
\begin{aligned}
G_{1}(p, t)= & \left(1+\frac{1}{p}\right) \theta(t)-\alpha_{y}(t) \\
G_{2}(p, q, t)= & \frac{1}{q} \beta_{x}(t) \bar{N}_{x}(t)+\mu_{y}(t)+\alpha_{y}(t) \\
& -\frac{1}{p} \theta(t)-\mu_{x}(t)-\alpha_{x}(t), \\
G_{2}^{\prime}(p, q, t)= & \frac{1}{q} \beta_{x}(t) \underline{x}_{s}(t)+\mu_{y}(t)+\alpha_{y}(t)-\frac{1}{p} \theta(t) \\
& -\mu_{x}(t)-\alpha_{x}(t), \\
W_{1}(p, t)= & p y_{e}(t)-y_{i}(t) \\
W_{2}(q, t)= & q y_{i}(t)-x_{i}(t)
\end{aligned}
$$

where $\left(x_{s}, x_{i}, y_{s}, y_{e}, y_{i}\right)$ is any solution of system (3).

We use the following two results in order to investigate the long-time behavior of system (3).

Lemma 5. If there exist positive constants $p>0$ and $T_{1}>0$ such that $G_{1}(p, t)<0$ for all $t \geq T_{1}$, then there exists $T_{2} \geq T_{1}$ such that either $W_{1}(p, t)>0$ for all $t \geq T_{2}$ or $W_{1}(p, t) \leq 0$ for all $t \geq T_{2}$.

Proof. Suppose that there does not exist $T_{2} \geq T_{1}$ such that $W_{1}(p, t)>0$ for all $t \geq T_{2}$ or $W_{1}(p, t) \leq 0$ for all $t \geq T_{2}$ hold. Then there necessarily exists $s_{1} \geq T_{1}$ such that $W_{1}\left(p, s_{1}\right)=0$ and $\left.\left(d W_{1}(p, t) / d t\right)\right|_{t=s_{1}}<0$. Hence we have

$$
p y_{e}\left(s_{1}\right)=y_{i}\left(s_{1}\right) \text {, }
$$




$$
\begin{aligned}
& \left.\frac{d W_{1}(p, t)}{d t}\right|_{t=s_{1}} \\
& =p \beta_{y}\left(s_{1}\right) x_{i}\left(s_{1}\right) y_{s}\left(s_{1}\right) \\
& \quad-p\left(\mu_{y}\left(s_{1}\right)+\theta\left(s_{1}\right)\right) y_{e}\left(s_{1}\right)-\theta\left(s_{1}\right) y_{e}\left(s_{1}\right) \\
& \quad+\left(\mu_{y}\left(s_{1}\right)+\alpha_{y}\left(s_{1}\right)\right) y_{i}\left(s_{1}\right) \\
& > \\
& \quad\left(\mu_{y}\left(s_{1}\right)+\alpha_{y}\left(s_{1}\right)\right) y_{i}\left(s_{1}\right) \\
& \quad-\left[\mu_{y}\left(s_{1}\right)+\left(1+\frac{1}{p}\right) \theta\left(s_{1}\right)\right] p y_{e}\left(s_{1}\right) .
\end{aligned}
$$

Substituting (27) into (28), we have

$$
\begin{aligned}
0 & >-\left\{\left(1+\frac{1}{p}\right) \theta\left(s_{1}\right)-\alpha_{y}\left(s_{1}\right)\right\} p y_{e}\left(s_{1}\right) \\
& =-p y_{e}\left(s_{1}\right) G_{1}\left(p, s_{1}\right) .
\end{aligned}
$$

From Lemma 4, we have $G_{1}\left(p, s_{1}\right)>0$, which is a contradiction.

Lemma 6. If there exist positive constants $p>0, q_{1}>$ $0, q_{2}>0$, and $T_{1}>0$ such that $G_{1}(p, t)<0, G_{2}\left(p, q_{1}, t\right)<$ $0, G_{2}^{\prime}\left(p, q_{2}, t\right)>0$ for all $t \geq T_{1}$, then there exists $T_{2} \geq T_{1}$ such that for every $t \geq T_{2}$, we have either $W_{2}\left(q_{i}, t\right)>0$ or $W_{2}\left(q_{i}, t\right) \leq 0, i=1,2$.

Proof. From Lemma 5, we know that there exists $T_{2}^{\prime} \geq T_{1}$ such that either $W_{1}(p, t)>0$ for all $t \geq T_{2}^{\prime}$ or $W_{1}(p, t) \leq 0$ for all $t \geq T_{2}^{\prime}$.

For the first case, if $W_{1}(p, t)>0$ for all $t \geq T_{2}^{\prime}$, we will show that there exists $T_{3} \geq T_{1}$ such that either $W_{2}\left(q_{1}, t\right)>0$ for all $t \geq T_{3}$ or $W_{2}\left(q_{1}, t\right) \leq 0$ for all $t \geq T_{3}$. Suppose that there does not exist $T_{3} \geq T_{1}$ such that $W_{2}\left(q_{1}, t\right)>0$ for all $t \geq T_{3}$ or $W_{2}\left(q_{1}, t\right) \leq 0$ for all $t \geq T_{3}$ hold. Then there necessarily exists $s_{2} \geq T_{1}$ such that $W_{2}\left(q_{1}, s_{2}\right)=0$ and $\left.\left(d W_{2}\left(q_{1}, t\right) / d t\right)\right|_{t=s_{2}}<0$. Hence we have

$$
\begin{aligned}
& q_{1} y_{i}\left(s_{2}\right)=x_{i}\left(s_{2}\right), \\
& \left.\frac{d W_{2}\left(q_{1}, t\right)}{d t}\right|_{t=s_{2}}=q_{1} \theta\left(s_{2}\right) y_{e}\left(s_{2}\right)-\left(\mu_{y}\left(s_{2}\right)\right. \\
& \left.\quad+\alpha_{y}\left(s_{2}\right)\right) q_{1} y_{i}\left(s_{2}\right)-\beta_{x}\left(s_{2}\right) x_{s}\left(s_{2}\right) y_{i}\left(s_{2}\right) \\
& +\left(\mu_{x}\left(s_{2}\right)+\alpha_{x}\left(s_{2}\right)\right) x_{i}\left(s_{2}\right) \geq \frac{q_{1}}{p} \theta\left(s_{2}\right) y_{i}\left(s_{2}\right) \\
& \quad-\left(\mu_{y}\left(s_{2}\right)+\alpha_{y}\left(s_{2}\right)\right) q_{1} y_{i}\left(s_{2}\right)-\beta_{x}\left(s_{2}\right) x_{s}\left(s_{2}\right) \\
& \cdot y_{i}\left(s_{2}\right)+\left(\mu_{x}\left(s_{2}\right)+\alpha_{x}\left(s_{2}\right)\right) x_{i}\left(s_{2}\right)=q_{1} y_{i}\left(s_{2}\right) \\
& \quad \cdot\left\{\frac{1}{p} \theta\left(s_{2}\right)-\mu_{y}\left(s_{2}\right)-\alpha_{y}\left(s_{2}\right)\right. \\
& \left.\quad-\frac{1}{q_{1}} \beta_{x}\left(s_{2}\right) x_{s}\left(s_{2}\right)\right\}+\left(\mu_{x}\left(s_{2}\right)+\alpha_{x}\left(s_{2}\right)\right) x_{i}\left(s_{2}\right) .
\end{aligned}
$$

From Lemma 4, we have $G_{2}^{\prime}\left(p, q_{2}, s_{3}\right)<0$, which is a contradiction. hold.

If we let $T_{2}=\max \left\{T_{3}, T_{4}\right\}$, then the results of the lemma

\section{Extinction of Infectious Population}

In this section, we obtain sufficient conditions for the extinction of the infectious population of system (3). The definition of the extinction is as follows. 
Definition 7. We say that the infective $x_{i}(t)$ of system (3) is extinct if

$$
\lim _{t \rightarrow+\infty} x_{i}(t)=0
$$

Similarly, we can define the extinction of $y_{i}(t)$. Next, one of the main results of this paper is investigated.

Theorem 8. If there exist positive constants $\lambda>0, p>0, q>$ 0 , and $T_{1}>0$ such that

$$
\begin{aligned}
& R_{1}(\lambda, p) \\
& :=\limsup _{t \rightarrow+\infty} \int_{t}^{t+\lambda}\left\{\frac{1}{p} \theta(s)-\mu_{y}(s)-\alpha_{y}(s)\right\} d s<0, \\
& R_{1}^{\prime}(\lambda, q) \\
& :=\limsup _{t \rightarrow+\infty} \int_{t}^{t+\lambda}\left\{\frac{1}{q} \beta_{x}(s) \bar{N}_{x}(s)-\mu_{x}(s)-\alpha_{x}(s)\right\} d s \\
& <0, \\
& R_{1}^{*}(\lambda, p, q) \\
& :=\limsup _{t \rightarrow+\infty} \int_{t}^{t+\lambda}\left\{p q \beta_{y}(s) \bar{N}_{y}(s)-\mu_{y}(s)-\theta(s)\right\} d s \\
& <0
\end{aligned}
$$

and $G_{1}(p, t)<0, G_{2}(p, q, t)<0$ for all $t \geq T_{1}$, then $\lim _{t \rightarrow+\infty} x_{i}(t)=0$ and $\lim _{t \rightarrow+\infty} y_{i}(t)=0$.

Proof. Since there exist $p>0, T_{1}>0$ such that $G_{1}(p, t)<0$ holds for all $t \geq T_{1}$, from Lemma 5 , we only have to consider the following two cases:

(i) $p y_{e}(t) \leq y_{i}(t)$ for all $t \geq T_{2}$.

(ii) $p y_{e}(t)>y_{i}(t)$ for all $t \geq T_{2}$.

First we consider case (i). Since we have $p y_{e}(t) \leq y_{i}(t)$ for all $t \geq T_{2}$, it follows from the fifth equation of system (3) that

$$
\begin{aligned}
\frac{d y_{i}(t)}{d t} & =\theta(t) y_{e}(t)-\mu_{y}(t) y_{i}(t)-\alpha_{y}(t) y_{i}(t) \\
& \leq\left\{\frac{1}{p} \theta(t)-\mu_{y}(t)-\alpha_{y}(t)\right\} y_{i}(t)
\end{aligned}
$$

for all $t \geq T_{2}$. Hence we have

$$
y_{i}(t) \leq y_{i}\left(T_{2}\right) \exp \left(\int_{T_{2}}^{t}\left\{\frac{1}{p} \theta(s)-\mu_{y}(s)-\alpha_{y}(s)\right\}\right)
$$

for all $t \geq T_{2}$. Now it follows from (37) that there exist constants $\delta_{1}>0$ and $T_{3}>T_{2}$ such that

$$
\int_{t}^{t+\lambda}\left\{\frac{1}{p} \theta(s)-\mu_{y}(s)-\alpha_{y}(s)\right\} d s<-\delta_{1}
$$

for all $t \geq T_{3}$. From (41) and (42) we have $\lim _{t \rightarrow+\infty} y_{i}(t)=0$. Then the second equation of the corresponding limit system
(3) is $d x_{i}(t) / d t=-\left(\mu_{x}(t)+\alpha_{x}(t)\right) x_{i}(t)$. From (i) and (H2) we can easily see that $\lim _{t \rightarrow+\infty} x_{i}(t)=0$.

Next we consider case (ii). Because there exist $p>0, q>0$ such that $G_{2}(p, q, t)<0$ for all $t \geq T_{1}$, then from the proof of Lemma 6, we know that there exists $T_{2}^{\prime} \geq \max \left\{T_{1}, T_{2}\right\}$ such that either $W_{2}(q, t)>0$ for all $t \geq T_{2}^{\prime}$ or $W_{2}(q, t) \leq 0$ for all $t \geq T_{2}^{\prime}$. Thus we also have two cases to discuss.

On the one hand, if $W_{2}(q, t) \leq 0$ holds for all $t \geq T_{2}^{\prime}$, that is, $y_{i}(t) \leq(1 / q) x_{i}(t)$ holds for all $t \geq T_{2}^{\prime}$, then from the second equation of system (3) we obtain

$$
\begin{aligned}
\frac{d x_{i}(t)}{d t} & =\beta_{x}(t) x_{s}(t) y_{i}(t)-\left(\mu_{x}(t)+\alpha_{x}(s)\right) x_{i}(t) \\
& \leq\left\{\frac{1}{q} \beta_{x}(t) \bar{N}_{x}(t)-\mu_{x}(t)-\alpha_{x}(t)\right\} x_{i}(t)
\end{aligned}
$$

for all $t \geq T_{2}^{\prime}$. Hence, we get

$$
\begin{aligned}
x_{i}(t) & \leq x_{i}\left(T_{2}^{\prime}\right) \\
\cdot \exp & \left(\int_{T_{2}^{\prime}}^{t}\left\{\frac{1}{q} \beta_{x}(s) \bar{N}_{x}(s)-\mu_{x}(s)-\alpha_{x}(s)\right\} d s\right)
\end{aligned}
$$

for all $t \geq T_{2}^{\prime}$. From (38) we see that there exist constants $\delta_{2}>$ 0 and $T_{4}>T_{2}^{\prime}$ such that

$$
\int_{t}^{t+\lambda}\left\{\frac{1}{q} \beta_{x}(s) \bar{N}_{x}(s)-\mu_{x}(s)-\alpha_{x}(s)\right\} d s<-\delta_{2}
$$

for all $t \geq T_{4}$. From (44) and (45), we have $\lim _{t \rightarrow+\infty} x_{i}(t)=0$.

On the other hand, if $W_{2}(q, t)>0$ for all $t \geq T_{2}^{\prime}$ hold, then we have $x_{i}(t)<q y_{i}(t)<p q y_{e}(t)$ for all $t \geq T_{2}^{\prime}$. From the fourth equation of system (3), we have

$$
\begin{aligned}
\frac{d y_{e}(t)}{d t} & =\beta_{y}(t) x_{i}(t) y_{s}(t)-\left(\mu_{y}(t)+\theta(t)\right) y_{e}(t) \\
& <\left\{p q \beta_{y}(t) \bar{N}_{y}(t)-\mu_{y}(t)-\theta(t)\right\} y_{e}(t)
\end{aligned}
$$

for all $t \geq T_{2}^{\prime}$. Hence we have

$$
\begin{aligned}
& y_{e}(t)<y_{e}\left(T_{2}^{\prime}\right) \\
& \quad \cdot \exp \left(\int_{T_{2}^{\prime}}^{t}\left\{p q \beta_{y}(s) \bar{N}_{y}(s)-\mu_{y}(s)-\theta(s)\right\}\right) .
\end{aligned}
$$

From (39), we see that there exist constants $\delta_{3}>0$ and $T_{5}>$ $T_{2}^{\prime}$ such that

$$
\int_{t}^{t+\lambda}\left\{p q \beta_{y}(s) \bar{N}_{y}(s)-\mu_{y}(s)-\theta(s)\right\} d s<-\delta_{3}
$$

for all $t \geq T_{5}$. From (47) and (48), we have $\lim _{t \rightarrow+\infty} y_{e}(t)=0$. Thus the fifth equation of the corresponding limit system (3) is $d y_{i}(t) / d t=-\left(\mu_{y}(t)+\alpha_{y}(t)\right) y_{i}(t)$. From (i) and (H2), we can easily get that $\lim _{t \rightarrow+\infty} y_{i}(t)=0$. In a similar way, we can also prove $\lim _{t \rightarrow+\infty} x_{i}(t)=0$. 
Remark 9. We assume that all coefficients of system (3) are given by identically constant functions; then system (3) becomes an autonomous system. Conditions (37)-(39) are as follows:

$$
\begin{array}{r}
\frac{1}{p} \theta-\mu_{y}-\alpha_{y}<0, \\
\frac{1}{q} \beta_{x} \frac{A_{x}}{\mu_{x}}-\mu_{x}-\alpha_{x}<0, \\
p q \beta_{y} \frac{A_{y}}{\mu_{y}}-\mu_{y}-\theta<0 .
\end{array}
$$

These inequalities show that $\theta \beta_{x} A_{x} / \mu_{x}\left(\mu_{y}+\alpha_{y}\right)\left(\mu_{x}+\alpha_{x}\right)<$ $p q<\mu_{y}\left(\mu_{y}+\theta\right) / \beta_{y} A_{y}$, which implies $R_{0}<1$; here $R_{0}$ is given in (2). This shows that the result is consistent with the literature [10].

Remark 10. It is obvious that if there exist positive constants $p>0, q>0$, and $T_{1}>0$ such that

$$
\begin{array}{r}
\limsup _{t \rightarrow+\infty}\left\{\frac{1}{p} \theta(t)-\mu_{y}(t)-\alpha_{y}(t)\right\}<0, \\
\limsup _{t \rightarrow+\infty}\left\{\frac{1}{q} \beta_{x}(t) \bar{N}_{x}(t)-\mu_{x}(t)-\alpha_{x}(t)\right\}<0, \\
\limsup _{t \rightarrow+\infty}\left\{p q \beta_{y}(t) \bar{N}_{y}(t)-\mu_{y}(t)-\theta(t)\right\}<0
\end{array}
$$

and $G_{1}(p, t)<0, G_{2}(p, q, t)<0$ for all $t \geq T_{1}$, then $\lim _{t \rightarrow+\infty} x_{i}(t)=0$ and $\lim _{t \rightarrow+\infty} y_{i}(t)=0$.

\section{Permanence of Infectious Population}

In this section, we obtain sufficient conditions for the permanence of infectious population of system (3).

The definition of the permanence is as follows.

Definition 11. We say that the infective $x_{i}(t)$ of system (3) is permanent if there exist positive constants $x_{i 1}>0$ and $x_{i 2}>0$, which are independent of the choice of initial value satisfying (3), such that

$$
0<x_{i 1} \leq \liminf _{t \rightarrow+\infty} x_{i}(t) \leq \limsup _{t \rightarrow+\infty} x_{i}(t) \leq x_{i 2} .
$$
$y_{i}(t)$.

Similarly, we can give the definition of the permanence of Let the function

$$
\begin{aligned}
& b\left(t, u_{1}, u_{2}\right):=3 \sqrt[3]{\beta_{x}(t) \beta_{y}(t) \theta(t) u_{1}(t) u_{2}(t)} \\
& -\left[\left(\mu_{x}(t)+\alpha_{x}(t)\right)+\left(\mu_{y}(t)+\alpha_{y}(t)\right)\right. \\
& \left.\quad+\left(\mu_{y}(t)+\theta(t)\right)\right]
\end{aligned}
$$

and let $\underline{N}_{x}(t)$ be some fixed solution of (8) with initial value $\underline{N}_{x}(0)>0$ and $\underline{N}_{y}(t)$ be some fixed solution of (10) with initial value $\underline{N}_{y}(0)>0$.
We have the following theorem.

Theorem 12. If there is a constant $\lambda_{1}>0$ such that

$$
R_{0}^{*}=\liminf _{t \rightarrow+\infty} \int_{t}^{t+\lambda_{1}} b\left(s, \underline{N}_{x}(s), \underline{N}_{y}(s)\right) d s>0,
$$

then the infective $x_{i}(t)$ and $y_{i}(t)$ of system (3) are permanent.

Proof. Since $\underline{N}_{x}(t) \leq N_{x}(t), \underline{N}_{y}(t) \leq N_{y}(t)$ for all $t \geq 0$, then, from system (3), we have

$$
\begin{aligned}
\frac{d x_{i}(t)}{d t} \geq & \beta_{x}(t)\left(\underline{N}_{x}(t)-x_{i}(t)\right) y_{i}(t)-\mu_{x}(t) x_{i}(t) \\
& -\alpha_{x}(t) x_{i}(t), \\
\frac{d y_{e}(t)}{d t} \geq & \beta_{y}(t)\left(\underline{N}_{y}(t)-y_{e}(t)-y_{i}(t)\right) x_{i}(t) \\
& -\left(\mu_{y}(t)+\theta(t)\right) y_{e}(t), \\
\frac{d y_{i}(t)}{d t}= & \theta(t) y_{e}(t)-\mu_{y}(t) y_{i}(t)-\alpha_{y}(t) y_{i}(t) .
\end{aligned}
$$

Let us consider the following system:

$$
\begin{aligned}
\frac{d \bar{x}_{i}(t)}{d t}= & \beta_{x}(t)\left(\underline{N}_{x}(t)-\bar{x}_{i}(t)\right) \bar{y}_{i}(t)-\mu_{x}(t) \bar{x}_{i}(t) \\
& -\alpha_{x}(t) \bar{x}_{i}(t) \\
\frac{d \bar{y}_{e}(t)}{d t}= & \beta_{y}(t)\left(\underline{N}_{y}(t)-\bar{y}_{e}(t)-\bar{y}_{i}(t)\right) \bar{x}_{i}(t) \\
& -\left(\mu_{y}(t)+\theta(t)\right) \bar{y}_{e}(t) \\
\frac{d \bar{y}_{i}(t)}{d t}= & \theta(t) \bar{y}_{e}(t)-\mu_{y}(t) \bar{y}_{i}(t)-\alpha_{y}(t) \bar{y}_{i}(t) .
\end{aligned}
$$

Firstly, we prove that the number $R_{0}^{*}$ is independent of the choice of $\underline{N}_{x}(t)$ and $\underline{N}_{y}(t)$. In fact, (c) of Lemma 3 implies that, for any sufficiently small $\epsilon>0\left(<\min \left(m_{1}, m_{2}\right)\right)$, any solution $\underline{N}_{x}^{*}(t)$ of (8) with initial value $\underline{N}_{x}^{*}(0)>0$, and any solution $\underline{N}_{y}^{*}(t)$ of (10) with initial value $\underline{N}_{y}^{*}(0)>0$, there exists $T>0$ such that as $t \geq T$

$$
\begin{aligned}
\underline{N}_{x}(t)-\epsilon & \leq \underline{N}_{x}^{*}(t) \leq \underline{N}_{x}(t)+\epsilon, \\
\underline{N}_{y}(t)-\epsilon & \leq \underline{N}_{y}^{*}(t) \leq \underline{N}_{y}(t)+\epsilon, \\
\underline{N}_{x}(t) & \geq m_{1}, \\
\underline{N}_{y}(t) & \geq m_{2} .
\end{aligned}
$$

Hence,

$$
\begin{aligned}
& b\left(t, \underline{N}_{x}(t)-\epsilon, \underline{N}_{y}(t)-\epsilon\right) \leq b\left(t, \underline{N}_{x}^{*}(t), \underline{N}_{y}^{*}(t)\right) \\
& \quad \leq b\left(t, \underline{N}_{x}(t)+\epsilon, \underline{N}_{y}(t)+\epsilon\right) .
\end{aligned}
$$


For $t \geq T$, since the inequality $\sqrt[3]{a_{1}+a_{2}+a_{3}} \leq \sqrt[3]{a_{1}}+\sqrt[3]{a_{2}}+$ $\sqrt[3]{a_{3}}$ holds for all $a_{i} \geq 0(i=1,2,3)$ and from Taylor's theorem of binary function, we obtain

$$
\begin{aligned}
\liminf _{t \rightarrow+\infty} \int_{t}^{t+\lambda_{1}} b\left(s, \underline{N}_{x}(s)+\epsilon, \underline{N}_{y}(s)+\epsilon\right) d s \leq R_{0}^{*} \\
+\left(\sqrt[3]{\left(M_{1}+M_{2}\right) \epsilon}+\sqrt[3]{\epsilon^{2}}\right) \\
\cdot 3 \lambda_{1} \sup _{t \geq 0} \sqrt[3]{\beta_{x}(t) \beta_{y}(t) \theta(t)}, \\
\liminf _{t \rightarrow+\infty} \int_{t}^{t+\lambda_{1}} b\left(s, \underline{N}_{x}(s)-\epsilon, \underline{N}_{y}(s)-\epsilon\right) d s \geq R_{0}^{*} \\
\quad-\epsilon \lambda_{1}\left(\sqrt[3]{\frac{M_{2}}{m_{1}^{2}}}+\sqrt[3]{\frac{M_{1}}{m_{2}^{2}}}\right) \sup _{t \geq 0} \sqrt[3]{\beta_{x}(t) \beta_{y}(t) \theta(t) .}
\end{aligned}
$$

By the arbitrariness of $\epsilon$, we finally obtain

$$
\liminf _{t \rightarrow+\infty} \int_{t}^{t+\lambda_{1}} b\left(s, \underline{N}_{x}^{*}(s), \underline{N}_{y}^{*}(s)\right) d s=R_{0}^{*} .
$$

This shows that $R_{0}^{*}$ is independent of the choice of $\underline{N}_{x}(t)$ and $\underline{N}_{y}(t)$. From inequalities (15) and (16), we can set $T$ largely enough such that

$$
\begin{aligned}
& m_{1}<\underline{N}_{x}(t) \leq N_{x}(t) \leq \bar{N}_{x}(t)<M_{1}, \\
& m_{2}<\underline{N}_{y}(t) \leq N_{y}(t) \leq \bar{N}_{y}(t)<M_{2}
\end{aligned}
$$

for all $t \geq T$. From (53), for sufficiently small $\eta>0$, there exists $T_{1} \geq T$ such that

$$
\int_{t}^{t+\lambda_{1}} b\left(s, \underline{N}_{x}(s), \underline{N}_{y}(s)\right) d s>\eta
$$

for all $t \geq T_{1}$. We define

$$
\begin{aligned}
& \beta_{x}^{+}:=\sup _{t \geq 0} \beta_{x}(t), \\
& \mu_{x}^{+}:=\sup _{t \geq 0} \mu_{x}(t), \\
& \beta_{y}^{+}:=\sup _{t \geq 0} \beta_{y}(t), \\
& \mu_{y}^{+}:=\sup _{t \geq 0} \mu_{y}(t), \\
& \alpha_{x}^{+}:=\sup _{t \geq 0} \alpha_{x}(t), \\
& \theta^{+}:=\sup _{t \geq 0} \theta(t), \\
& \alpha_{y}^{+}:=\sup _{t \geq 0} \alpha_{y}(t) .
\end{aligned}
$$

From (60)-(63), we see that, for positive constants $\eta_{1}<\eta$, there exist small $\epsilon_{i}>0,(i=1,2,3)$ and $T_{2} \geq T_{1}$ such that

$$
\begin{aligned}
& \int_{t}^{t+\lambda_{1}} b\left(s, \underline{N}_{x}(s)-\epsilon_{1}-\kappa_{1} \epsilon_{2}, \underline{N}_{y}(s)-\kappa_{2} \epsilon_{1}-\kappa_{3} \epsilon_{2}\right. \\
& \left.\quad-\epsilon_{3}\right) d s>\eta_{1} \\
& \underline{N}_{x}(t)-\epsilon_{1}-\kappa_{1} \epsilon_{2}>m_{1}, \\
& \underline{N}_{y}(t s)-\kappa_{2} \epsilon_{1}-\kappa_{3} \epsilon_{2}-\epsilon_{3}>m_{2}
\end{aligned}
$$

hold for all $t \geq T_{2}$, where $\kappa_{1}:=\beta_{x}^{+} M_{1} \omega_{3}, \kappa_{2}:=$ $\beta_{y}^{+} M_{2} \omega_{4}, \kappa_{3}:=1+\kappa_{1} \kappa_{2}$. From (H2), we can choose $\epsilon_{1}, \epsilon_{2}$ such that both the following two equations hold for all $t \geq T_{2}$.

$$
\begin{aligned}
& \int_{t}^{t+\omega_{3}}\left\{\beta_{x}(s) M_{1} \epsilon_{2}-\left(\mu_{x}(s)+\alpha_{x}(s)\right) \epsilon_{1}\right\} d s<-\eta_{1}, \\
& \int_{t}^{t+\omega_{4}}\left\{\beta_{y}(s) M_{2}\left(\epsilon_{1}+\kappa_{1} \epsilon_{2}\right)-\left(\mu_{y}(s)+\theta(s)\right) \epsilon_{3}\right\} d s \\
& \quad<-\eta_{1} .
\end{aligned}
$$

First we claim that $\lim \sup _{t \rightarrow+\infty} \bar{y}_{i}(t)>\epsilon_{2}$. In fact, if it is not true, then there exists $T_{3} \geq T_{2}$ such that

$$
\bar{y}_{i}(t) \leq \epsilon_{2}
$$

for all $t \geq T_{3}$. Suppose that $\bar{x}_{i}(t) \geq \epsilon_{1}$ for all $t \geq T_{3}$. Then, from (60) and (69) we have

$$
\begin{gathered}
\bar{x}_{i}(t)=\bar{x}_{i}\left(T_{3}\right)+\int_{T_{3}}^{t}\left\{\beta_{x}(s)\left(\underline{N}_{x}(s)-\bar{x}_{i}(s)\right) \bar{y}_{i}(s)\right. \\
\left.-\left(\mu_{x}(s)+\alpha_{x}(s)\right) \bar{x}_{i}(s)\right\} d s \leq \bar{x}_{i}\left(T_{3}\right) \\
+\int_{T_{3}}^{t}\left\{\beta_{x}(s) M_{1} \epsilon_{2}-\left(\mu_{x}(s)+\alpha_{x}(s)\right) \epsilon_{1}\right\} d s
\end{gathered}
$$

for all $t \geq T_{3}$. Thus, from (67), we have $\lim _{t \rightarrow+\infty} \bar{x}_{i}(t)=-\infty$, which contradicts with (b) of Lemma 3. Therefore we see that there exist $s_{1} \geq T_{3}$ such that $\bar{x}_{i}\left(s_{1}\right)<\epsilon_{1}$. Suppose that there exists $s_{2} \geq s_{1}$ such that $\bar{x}_{i}\left(s_{2}\right)>\epsilon_{1}+\kappa_{1} \epsilon_{2}$. Then, we see that there necessarily exists $s_{3} \in\left(s_{1}, s_{2}\right)$ such that $\bar{x}_{i}\left(s_{3}\right)=\epsilon_{1}$ and $\bar{x}_{i}(t)>\epsilon_{1}$ for all $t \in\left(s_{3}, s_{2}\right]$. It is easy to see that there exists an integer $n$ that satisfies $s_{2} \in\left[s_{3}+n \omega_{3}, s_{3}+(n+1) \omega_{3}\right)$. Then, from (67), we have

$$
\begin{aligned}
\epsilon_{1} & +\kappa_{1} \epsilon_{2}<\bar{x}_{i}\left(s_{2}\right)=\bar{x}_{i}\left(s_{3}\right) \\
& +\int_{s_{3}}^{s_{2}}\left\{\beta_{x}(s)\left(\underline{N}_{x}(s)-\bar{x}_{i}(s)\right) \bar{y}_{i}(s)\right. \\
& \left.-\left(\mu_{x}(s)+\alpha_{x}(s)\right) \bar{x}_{i}(s)\right\} d s<\epsilon_{1}+\left\{\int_{s_{3}}^{s_{3}+n \omega_{3}}\right. \\
& \left.+\int_{s_{3}+n \omega_{3}}^{s_{2}}\right\}\left\{\beta_{x}(s) M_{1} \epsilon_{2}-\left(\mu_{x}(s)+\alpha_{x}(s)\right) \epsilon_{1}\right\} d s \\
& \leq \epsilon_{1}+\int_{s_{3}+n \omega_{3}}^{s_{2}} \beta_{x}(s) M_{1} \epsilon_{2} d s<\epsilon_{1}+\kappa_{1} \epsilon_{2},
\end{aligned}
$$


which is a contradiction. Therefore, we see that

$$
\bar{x}_{i}(t) \leq \epsilon_{1}+\kappa_{1} \epsilon_{2}
$$

for all $t \geq s_{1}$. In a similar way, from (68), we can show that there exists $\widetilde{s_{1}} \geq s_{1}$ such that

$$
\bar{y}_{e}(t) \leq \kappa_{2} \epsilon_{1}+\kappa_{1} \kappa_{2} \epsilon_{2}+\epsilon_{3}
$$

for all $t \geq \widetilde{s_{1}}$.

For $t \geq 0$, we define a differentiable function $V(t)=$ $\bar{x}_{i}(t) \bar{y}_{e}(t) \bar{y}_{i}(t)$. When $t \geq \widetilde{s_{1}}$, from (69)-(73), we have

$$
\begin{aligned}
& \left.\frac{d V(t)}{d t}\right|_{(55)} \\
& =\beta_{x}(t)\left(\underline{N}_{x}(t)-\bar{x}_{i}(t)\right) \bar{y}_{e}(t) \bar{y}_{i}^{2}(t) \\
& \quad+\beta_{y}(t)\left(\underline{N}_{y}(t)-\bar{y}_{e}(t)-\bar{y}_{i}(t)\right) \bar{x}_{i}^{2}(t) \bar{y}_{i}(t) \\
& \quad+\theta(t) \bar{x}_{i}(t) \bar{y}_{e}^{2}(t)
\end{aligned}
$$

$$
\begin{aligned}
& -\left(\mu_{x}(t)+2 \mu_{y}(t)+\theta(t)+\alpha_{x}(t)+\alpha_{y}(t)\right) V(t) \\
\geq & \beta_{x}(t)\left(\underline{N}_{x}(t)-\epsilon_{1}-\kappa_{1} \epsilon_{2}\right) \bar{y}_{e}(t) \bar{y}_{i}^{2}(t) \\
& +\beta_{y}(t)\left(\underline{N}_{y}(s)-\kappa_{2} \epsilon_{1}-\kappa_{3} \epsilon_{2}-\epsilon_{3}\right) \bar{x}_{i}^{2}(t) \bar{y}_{i}(t) \\
& +\theta(t) \bar{x}_{i}(t) \bar{y}_{e}^{2}(t) \\
& -\left(\mu_{x}(t)+2 \mu_{y}(t)+\theta(t)+\alpha_{x}(t)+\alpha_{y}(t)\right) V(t) .
\end{aligned}
$$

Since the inequality $a+b+c \geq 3 \sqrt[3]{a b c}$ holds for all $a, b, c \geq 0$ and from (65) and (66), we have

$$
\begin{aligned}
& \left.\frac{d V(t)}{d t}\right|_{(55)} \geq b\left(t, \underline{N}_{x}(t)-\epsilon_{1}-\kappa_{1} \epsilon_{2}, \underline{N}_{y}(t)-\kappa_{2} \epsilon_{1}\right. \\
& \left.-\kappa_{3} \epsilon_{2}-\epsilon_{3}\right) V(t) .
\end{aligned}
$$

Integrating the above inequality from $\widetilde{s_{1}}$ to $t$, we have

$$
V(t) \geq V\left(\widetilde{s_{1}}\right) \exp \left(\int_{\widetilde{s_{1}}}^{t} b\left(s, \underline{N}_{x}(s)-\epsilon_{1}-\kappa_{1} \epsilon_{2}, \underline{N}_{y}(s)-\kappa_{2} \epsilon_{1}-\kappa_{3} \epsilon_{2}-\epsilon_{3}\right) d s\right)
$$

for all $t \geq \widetilde{s_{1}}$. By (62), we obtain $\lim _{t \rightarrow+\infty} V(t)=\infty$. This contradicts with the boundedness of $\bar{x}_{i}(t), \bar{y}_{e}(t)$, and $\bar{y}_{i}(t)$. From this contradiction, we finally conclude $\lim \sup _{t \rightarrow+\infty} \bar{y}_{i}(t)>$ $\epsilon_{2}$.

Next, we prove

$$
\liminf _{t \rightarrow+\infty} \bar{y}_{i}(t) \geq \bar{y}_{i 1},
$$

where $\bar{y}_{i 1}>0$ is a constant given in the following lines. For convenience sake, we let $\omega^{*}$ be the least common multiple of $\omega_{3}, \omega_{4}$, and $\lambda_{1}$. If we define

$$
\begin{aligned}
\liminf _{t \rightarrow+\infty} b\left(t, \underline{N}_{x}(t)-\epsilon_{1}-\kappa_{1} \epsilon_{2}, \underline{N}_{y}(t)-\kappa_{2} \epsilon_{1}-\kappa_{3} \epsilon_{2}\right. \\
\left.-\epsilon_{3}\right):=\underline{m}
\end{aligned}
$$

for $\epsilon>0$, there exists $\widetilde{T_{3}}\left(\geq T_{2}\right)$ such that

$$
\begin{aligned}
& b\left(t, \underline{N}_{x}(t)-\epsilon_{1}-\kappa_{1} \epsilon_{2}, \underline{N}_{y}(t)-\kappa_{2} \epsilon_{1}-\kappa_{3} \epsilon_{2}-\epsilon_{3}\right) \\
& >\underline{m}-\epsilon
\end{aligned}
$$

for all $t \geq \widetilde{T_{3}}$. Then, from inequalities (64) and (67)-(68) and $(\mathrm{H} 2)$, we see that there exist constants $\widetilde{T_{4}}\left(\geq \widetilde{T_{3}}\right), \lambda_{2}(>$ $\left.\max \left(\omega_{5}, \omega_{6}\right)\right)$, here we choose $\lambda_{2}$ is an integral multiple of $\omega^{*}$, and $\eta_{2}>0$ such that

$$
\begin{aligned}
& \int_{t}^{t+\lambda_{3}}\left\{\beta_{x}(s) M_{1} \epsilon_{2}-\left(\mu_{x}(s)+\alpha_{x}(s)\right) \epsilon_{1}\right\} d s<-M_{1}, \\
& \int_{t}^{t+\lambda_{3}}\left\{\beta_{y}(s) M_{2}\left(\epsilon_{1}+\kappa_{1} \epsilon_{2}\right)-\left(\mu_{y}(s)+\theta(s)\right) \epsilon_{3}\right\} d s \\
& \quad<-M_{2},
\end{aligned}
$$

$$
\begin{aligned}
& \int_{t}^{t+\lambda_{3}} b\left(s, \underline{N}_{x}(s)-\epsilon_{1}-\kappa_{1} \epsilon_{2}, \underline{N}_{y}(s)-\kappa_{2} \epsilon_{1}-\kappa_{3} \epsilon_{2}\right. \\
& \left.\quad-\epsilon_{3}\right) d s>\eta_{2}, \\
& \int_{t}^{t+\lambda_{3}} \beta_{x}(s) d s>\eta_{2}, \\
& \int_{t}^{t+\lambda_{3}} \beta_{y}(s) d s>\eta_{2}
\end{aligned}
$$

for all $t \geq \widetilde{T_{4}}, \lambda_{3} \geq \lambda_{2}$, and $\lambda_{3}$ is an integral multiple of $\omega$. Let $K_{0}>0$ be a positive integer satisfying

$$
\begin{gathered}
e^{-\left(\mu_{x}^{+}+\alpha_{x}^{+}+\mu_{y}^{+}+\theta^{+}\right) \lambda_{2}} \eta_{2}^{2} m_{1} m_{2} \nu_{2} \nu_{3} e^{K_{0} \eta_{2}+(\underline{m}-\epsilon) \omega^{*}} \\
>\left(\epsilon_{1}+\kappa_{1} \epsilon_{2}\right)\left[\epsilon_{3}+\left(\epsilon_{1}+\kappa_{1} \epsilon_{2}\right) \kappa_{2}\right],
\end{gathered}
$$

where $\nu_{2}:=\epsilon_{2} e^{-3\left(\mu_{y}^{+}+\alpha_{y}^{+}\right) \lambda_{2}}, \nu_{3}:=e^{-2\left(\mu_{x}^{+}+\alpha_{x}^{+}\right) \lambda_{2}} \eta_{2} m_{1} \nu_{2}$. Since we have proved $\lim \sup _{t \rightarrow+\infty} y_{i}(t)>\epsilon_{2}$, there are only two possibilities as follows:

(i) There exists $\widetilde{T_{5}} \geq \widetilde{T_{4}}$ such that $\bar{y}_{i}(t) \geq \epsilon_{2}$ for all $t \geq \widetilde{T_{5}}$.

(ii) $\bar{y}_{i}(t)$ oscillates about $\epsilon_{2}$ for large $t \geq \widetilde{T_{4}}$.

In case (i), we have $\liminf _{t \rightarrow+\infty} \bar{y}_{i}(t) \geq \epsilon_{2}:=\bar{y}_{i 1}$. In case (ii), then there necessarily exist two constants $t_{1}, t_{2} \geq \widetilde{T_{4}}\left(t_{2} \geq t_{1}\right)$ such that

$$
\begin{aligned}
& \bar{y}_{i}\left(t_{1}\right)=\bar{y}_{i}\left(t_{2}\right)=\epsilon_{2}, \\
& \bar{y}_{i}(t)<\epsilon_{2}, \quad \forall t \in\left(t_{1}, t_{2}\right) .
\end{aligned}
$$


Suppose that $t_{2}-t_{1} \leq\left(K_{0}+3\right) \lambda_{2}$. Then, from the third equation of system (55), we have

$$
\frac{d \bar{y}_{i}(t)}{d t} \geq-\left(\mu_{y}^{+}+\alpha_{y}^{+}\right) \bar{y}_{i}(t)
$$

Hence, we obtain

$$
\begin{gathered}
\bar{y}_{i}(t) \geq \bar{y}_{i}\left(t_{1}\right) \exp \left(\int_{t_{1}}^{t}-\left(\mu_{y}^{+}+\alpha_{y}^{+}\right) d s\right) \\
\geq \epsilon_{2} e^{-\left(\mu_{y}^{+}+\alpha_{y}^{+}\right)\left(K_{0}+3\right) \lambda_{2}}:=\bar{y}_{i 1}
\end{gathered}
$$

for all $t \in\left(t_{1}, t_{2}\right)$. Suppose that $t_{2}-t_{1}>\left(K_{0}+3\right) \lambda_{2}$. Then, from (87), we have

$$
\bar{y}_{i}(t) \geq \epsilon_{2} e^{-\left(\mu_{y}^{+}+\alpha_{y}^{+}\right)\left(K_{0}+3\right) \lambda_{2}}=\bar{y}_{i 1}
$$

for all $t \in\left(t_{1}, t_{1}+\left(K_{0}+3\right) \lambda_{2}\right)$. Now, we are in a position to show that $\bar{y}_{i}(t) \geq \bar{y}_{i 1}$ for all $t \in\left[t_{1}+\left(K_{0}+3\right) \lambda_{2}, t_{2}\right)$. Suppose that $\bar{x}_{i}(t) \geq \epsilon_{1}$ for all $t \in\left[t_{1}, t_{1}+\lambda_{2}\right]$. Then, from (80), we have

$$
\begin{aligned}
& \bar{x}_{i}\left(t_{1}+\lambda_{2}\right) \\
& \leq \bar{x}_{i}\left(t_{1}\right) \\
& \quad+\int_{t_{1}}^{t_{1}+\lambda_{2}}\left\{\beta_{x}(s) M_{1} \epsilon_{2}-\left(\mu_{x}(s)+\alpha_{x}(s)\right) \epsilon_{1}\right\} d s \\
& <M_{1}-M_{1}=0,
\end{aligned}
$$

which is a contradiction. Therefore, there exists $s_{4} \in\left[t_{1}, t_{1}+\right.$ $\left.\lambda_{2}\right]$ such that $x_{i}\left(s_{4}\right)<\epsilon_{1}$. Then, similar to the proof that $\lim \sup _{t \rightarrow+\infty} \bar{y}_{i}(t)>\epsilon_{2}$, we can show that $\bar{x}_{i}(t) \leq \epsilon_{1}+\kappa_{1} \epsilon_{2}$ for all $t \geq s_{4}$. Similarly, from (75), we can show that there exists $\widetilde{s_{4}} \in\left[t_{1}, t_{1}+\lambda_{2}\right]$ such that $\bar{y}_{e}(t) \leq \epsilon_{3}+\kappa_{2} \epsilon_{1}+\kappa_{1} \kappa_{2} \epsilon_{2}$ for all $t \geq \widetilde{s_{4}}$. Thus we have

$$
\begin{aligned}
& \bar{x}_{i}(t) \leq \epsilon_{1}+\kappa_{1} \epsilon_{2}, \\
& \bar{y}_{e}(t) \leq \epsilon_{3}+\kappa_{2} \epsilon_{1}+\kappa_{1} \kappa_{2} \epsilon_{2}
\end{aligned}
$$

for all $t \geq t_{1}+\lambda_{2} \geq \max \left(s_{4}, \widetilde{s_{4}}\right)$. From (87), we have

$$
\bar{y}_{i}(t) \geq \nu_{2}=\epsilon_{2} e^{-3\left(\mu_{y}^{+}+\alpha_{y}^{+}\right) \lambda_{2}}>\bar{y}_{i 1}
$$

for all $t \in\left[t_{1}, t_{1}+3 \lambda_{2}\right]$. Thus, from (65), (91), and (92), we have

$$
\begin{aligned}
\frac{d \bar{x}_{i}(t)}{d t}= & \beta_{x}(t)\left(\underline{N}_{x}(t)-\bar{x}_{i}(t)\right) \bar{y}_{i}(t) \\
& -\left(\mu_{x}(t)+\alpha_{x}(t)\right) \bar{x}_{i}(t) \\
\geq & \beta_{x}(t) m_{1} \nu_{2}-\left(\mu_{x}^{+}+\alpha_{x}^{+}\right) \bar{x}_{i}(t)
\end{aligned}
$$

for all $t \in\left[t_{1}+\lambda_{2}, t_{1}+3 \lambda_{2}\right]$. Hence, from (83), we obtain

$$
\begin{aligned}
& \bar{x}_{i}\left(t_{1}+3 \lambda_{2}\right) \\
& \geq e^{-\left(\mu_{x}^{+}+\alpha_{x}^{+}\right)\left(t_{1}+3 \lambda_{2}\right)}\left\{\bar{x}_{i}\left(t_{1}+2 \lambda_{2}\right) e^{\left(\mu_{x}^{+}+\alpha_{x}^{+}\right)\left(t_{1}+2 \lambda_{2}\right)}\right. \\
& \left.+\int_{t_{1}+2 \lambda_{2}}^{t_{1}+3 \lambda_{2}} e^{\left(\mu_{x}^{+}+\alpha_{x}^{+}\right) s} \beta_{x}(s) m_{1} \nu_{2} d s\right\} \\
& \geq e^{-\left(\mu_{x}^{+}+\alpha_{x}^{+}\right)\left(t_{1}+3 \lambda_{2}\right)} \int_{t_{1}+2 \lambda_{2}}^{t_{1}+3 \lambda_{2}} e^{\left(\mu_{x}^{+}+\alpha_{x}^{+}\right) s} \beta_{x}(s) m_{1} v_{2} d s \\
& \geq e^{-\left(\mu_{x}^{+}+\alpha_{x}^{+}\right) \lambda_{2}} \eta_{2} m_{1} \nu_{2} \\
& \bar{x}_{i}(t) \geq e^{-\left(\mu_{x}^{+}+\alpha_{x}^{+}\right) t}\left\{\bar{x}_{i}\left(t_{1}+\lambda_{2}\right) e^{\left(\mu_{x}^{+}+\alpha_{x}^{+}\right)\left(t_{1}+\lambda_{2}\right)}\right. \\
& \left.+\int_{t_{1}+\lambda_{2}}^{t} e^{\left(\mu_{x}^{+}+\alpha_{x}^{+}\right) s} \beta_{x}(s) m_{1} v_{2} d s\right\} \\
& \geq e^{-\left(\mu_{x}^{+}+\alpha_{x}^{+}\right)\left(t_{1}+3 \lambda_{2}\right)} \int_{t_{1}+\lambda_{2}}^{t_{1}+2 \lambda_{2}} e^{\left(\mu_{x}^{+}+\alpha_{x}^{+}\right) s} \beta_{x}(s) m_{1} v_{2} d s \\
& \geq e^{-2\left(\mu_{x}^{+}+\alpha_{x}^{+}\right) \lambda_{2}} \eta_{2} m_{1} \nu_{2}=\nu_{3}
\end{aligned}
$$

for all $t \in\left[t_{1}+2 \lambda_{2}, t_{1}+3 \lambda_{2}\right]$. Thus, from (66), (91), (92), and (95), we have

$$
\begin{aligned}
\frac{d \bar{y}_{e}(t)}{d t}= & \beta_{y}(t)\left(\underline{N}_{y}(t)-\bar{y}_{e}(t)-\bar{y}_{i}(t)\right) \bar{x}_{i}(t) \\
& -\left(\mu_{y}(t)+\theta(t)\right) \bar{y}_{e}(t) \\
\geq & \beta_{y}(t) m_{2} \nu_{3}-\left(\mu_{y}(t)+\theta(t)\right) \bar{y}_{e}(t)
\end{aligned}
$$

for all $t \in\left[t_{1}+2 \lambda_{2}, t_{1}+3 \lambda_{2}\right]$. Hence, from (84), we obtain

$$
\begin{aligned}
& \bar{y}_{e}\left(t_{1}+3 \lambda_{2}\right) \\
& \geq e^{-\left(\mu_{y}^{+}+\theta^{+}\right)\left(t_{1}+3 \lambda_{2}\right)}\left\{\bar{y}_{e}\left(t_{1}+2 \lambda_{2}\right) e^{\left(\mu_{y}^{+}+\theta^{+}\right)\left(t_{1}+2 \lambda_{2}\right)}\right. \\
& \left.\quad+\int_{t_{1}+2 \lambda_{2}}^{t_{1}+3 \lambda_{2}} \beta_{y}(s) m_{2} \nu_{3} e^{\left(\mu_{y}^{+}+\theta^{+}\right) s} d s\right\} \\
& \geq e^{-\left(\mu_{y}^{+}+\theta^{+}\right)\left(t_{1}+3 \lambda_{2}\right)} \int_{t_{1}+2 \lambda_{2}}^{t_{1}+3 \lambda_{2}} \beta_{y}(s) m_{2} \nu_{3} e^{\left(\mu_{y}^{+}+\theta^{+}\right) s} d s \\
& \geq e^{-\left(\mu_{y}^{+}+\theta^{+}\right) \lambda_{2}} \eta_{2} m_{2} \nu_{3} .
\end{aligned}
$$


We claim that $\bar{y}_{i}(t)>\bar{y}_{i 1}$ for all $t \in\left(t_{1}+\left(K_{0}+3\right) \lambda_{2}, t_{2}\right)$. If it is not true, there exists a $t_{0}>0$ such that $t_{0} \in\left(t_{1}+\left(K_{0}+\right.\right.$ 3) $\left.\lambda_{2}, t_{2}\right), \bar{y}_{i}\left(t_{0}\right)=\bar{y}_{i 1}$ and $\bar{y}_{i}(t) \geq \bar{y}_{i 1}$ for all $t \in\left(t_{1}, t_{0}\right)$. Then there exists $m \in N$ such that $t_{0} \in\left[t_{1}+\left(K_{0}+3\right) \lambda_{2}+m \omega, t_{1}+\right.$ $\left.\left(K_{0}+3\right) \lambda_{2}+(m+1) \omega\right)$. Let $V(t)=\bar{x}_{i}(t) \bar{y}_{e}(t) \bar{y}_{i}(t)$. From $(74)$, (75), (91), and (92), the derivative of $V(t)$ along solutions of (55) satisfies

$$
\begin{aligned}
& \left.\frac{d V(t)}{d t}\right|_{(55)} \geq b\left(t, \underline{N}_{x}(t)-\epsilon_{1}-\kappa_{1} \epsilon_{2}, \underline{N}_{y}(t)-\kappa_{2} \epsilon_{1}\right. \\
& \left.-\kappa_{3} \epsilon_{2}-\epsilon_{3}\right) V(t)
\end{aligned}
$$

for all $t \in\left[t_{1}+\lambda_{2}, t_{2}\right]$. Integrating the above inequality from $t_{1}+3 \lambda_{2}$ to $t_{0}$, we have

$$
\begin{aligned}
& \bar{x}_{i}\left(t_{0}\right) \bar{y}_{e}\left(t_{0}\right) \bar{y}_{i}\left(t_{0}\right) \geq \bar{x}_{i}\left(t_{1}+3 \lambda_{2}\right) \bar{y}_{e}\left(t_{1}+3 \lambda_{2}\right) \bar{y}_{i}\left(t_{1}+3 \lambda_{2}\right) \\
& \quad \cdot \exp \left(\int_{t_{1}+3 \lambda_{2}}^{t_{0}} b\left(t, \underline{N}_{x}(t)-\epsilon_{1}-\kappa_{1} \epsilon_{2}, \underline{N}_{y}(t)-\kappa_{2} \epsilon_{1}-\kappa_{3} \epsilon_{2}-\epsilon_{3}\right) d t\right) \geq e^{-\left(\mu_{x}^{+}+\alpha_{x}^{+}+\mu_{y}^{+}+\theta^{+}\right) \lambda_{2}} \eta_{2}^{2} m_{1} m_{2} \nu_{2} \nu_{3} \bar{y}_{i 1} \\
& \quad \cdot \exp \left(\left\{\int_{t_{1}+3 \lambda_{2}}^{t_{1}+\left(K_{0}+3\right) \lambda_{2}}+\int_{t_{1}+\left(K_{0}+3\right) \lambda_{2}}^{t_{1}+\left(K_{0}+3\right) \lambda_{2}+m \omega}+\int_{t_{1}+\left(K_{0}+3\right) \lambda_{2}+m \omega}^{t_{0}}\right\} b\left(t, \underline{N}_{x}(t)-\epsilon_{1}-\kappa_{1} \epsilon_{2}, \underline{N}_{y}(t)-\kappa_{2} \epsilon_{1}-\kappa_{3} \epsilon_{2}-\epsilon_{3}\right) d t\right) \\
& \quad \geq e^{-\left(\mu_{x}^{+}+\alpha_{x}^{+}+\mu_{y}^{+}+\theta^{+}\right) \lambda_{2}} \eta_{2}^{2} m_{1} m_{2} \nu_{2} \nu_{3} \bar{y}_{i 1} e^{K_{0} \eta_{2}+(\underline{m}-\epsilon) \omega^{*}}>\left(\epsilon_{1}+\kappa_{1} \epsilon_{2}\right)\left[\epsilon_{3}+\left(\epsilon_{1}+\kappa_{1} \epsilon_{2}\right) \kappa_{2}\right] \bar{y}_{i 1} .
\end{aligned}
$$

Thus, $\bar{x}_{i}\left(t_{0}\right) \bar{y}_{e}\left(t_{0}\right)>\left(\epsilon_{1}+\kappa_{1} \epsilon_{2}\right)\left[\epsilon_{3}+\left(\epsilon_{1}+\kappa_{1} \epsilon_{2}\right) \kappa_{2}\right]$, which contradicts with (91).

Therefore, $\bar{y}_{i}(t) \geq \bar{y}_{i 1}$ is valid for all $t \in\left[t_{1}, t_{2}\right]$, which implies $\liminf _{t \rightarrow+\infty} \bar{y}_{i}(t) \geq \bar{y}_{i 1}>0$. According to the comparison theorem, we have $\liminf _{t \rightarrow+\infty} y_{i}(t) \geq \bar{y}_{i 1}>0$. Since $\lim \sup _{t \rightarrow+\infty} y_{i}(t) \leq \lim \sup _{t \rightarrow+\infty} N_{y}(t) \leq M_{2}<+\infty$, the infective $y_{i}(t)$ of system (3) is permanent.

Furthermore, from system (3), there exists $x_{i 1}>0$ such that $\lim \inf _{t \rightarrow+\infty} x_{i}(t) \geq x_{i 1}>0$. Since $\lim \sup _{t \rightarrow+\infty} x_{i}(t) \leq$ $\lim \sup _{t \rightarrow+\infty} N_{x}(t) \leq M_{1}<+\infty$, the infective $x_{i}(t)$ of system (3) is also permanent. This completes the proof.

Remark 13. For the corresponding autonomous system of system (3), condition (53) can become as follows:

$$
\begin{aligned}
3 \sqrt[3]{\beta_{x} \beta_{y} \theta \frac{A_{x}}{\mu_{x}+\alpha_{x}} \frac{A_{y}}{\mu_{y}+\alpha_{y}}} \\
>\left(\mu_{x}+\alpha_{x}\right)+\left(\mu_{y}+\alpha_{y}\right)+\left(\mu_{y}+\theta\right) \\
\geq 3 \sqrt[3]{\left(\mu_{x}+\alpha_{x}\right)\left(\mu_{y}+\alpha_{y}\right)\left(\mu_{y}+\theta\right) .}
\end{aligned}
$$

This shows that $\sqrt[3]{\beta_{x} \beta_{y} \theta\left(A_{x} / \mu_{x}\right)\left(A_{y} / \mu_{y}\right)}>$ $\sqrt[3]{\left(\mu_{x}+\alpha_{x}\right)\left(\mu_{y}+\alpha_{y}\right)\left(\mu_{y}+\theta\right)}$, which implies $R_{0}>1$; here $R_{0}$ is given in (2). This means that the result is consistent with the literature [10].

Remark 14. It is easy to prove that if $\lim _{i n f} \operatorname{lit+\infty }_{t \rightarrow} b\left(t, \underline{N}_{x}(t)\right.$, $\left.\underline{N}_{y}(t)\right)>0$, then the infective $y_{i}(t)$ of system (3) is permanent.

\section{Some Corollaries}

As consequences of Theorems 8 and 12, we have a series of corollaries as follows.
Corollary 15. Suppose that $\mu_{x}(t)>0, \mu_{y}(t)>0$ for all $t \geq 0$ and $0<\liminf _{t \rightarrow \infty}\left(A_{x}(t) /\left(\mu_{x}(t)+\alpha_{x}(t)\right)\right) \leq$ $\lim \sup _{t \rightarrow \infty}\left(A_{x}(t) / \mu_{x}(t)\right)<\infty, 0<\liminf _{t \rightarrow \infty}\left(A_{y}(t) /\right.$ $\left.\left(\mu_{y}(t)+\alpha_{y}(t)\right)\right) \leq \lim \sup _{t \rightarrow \infty}\left(A_{y}(t) / \mu_{y}(t)\right)<\infty$. If there exist positive constants $\lambda>0, p>0, q>0$, and $T_{1}>0$ such that

$$
\begin{aligned}
& \limsup _{t \rightarrow+\infty} \int_{t}^{t+\lambda}\left\{\frac{1}{p} \theta(s)-\mu_{y}(s)-\alpha_{y}(s)\right\} d s<0, \\
& \underset{\limsup }{\operatorname{ta+\infty }} \int_{t}^{t+\lambda}\left\{\frac{1}{q} \beta_{x}(s)\left(\frac{A_{x}}{\mu_{x}}\right)^{M}-\mu_{x}(s)-\alpha_{x}(s)\right\} d s \\
& \quad<0,
\end{aligned}
$$

$$
\begin{aligned}
& \limsup _{t \rightarrow+\infty} \int_{t}^{t+\lambda}\left\{p q \beta_{y}(s)\left(\frac{A_{y}}{\mu_{y}}\right)^{M}-\mu_{y}(s)-\theta(s)\right\} d s \\
& <0
\end{aligned}
$$

and $G_{1}(p, t)<0, G_{2}(p, q, t)<0$ for all $t \geq T_{1}$, then the two infectious populations $x_{i}(t)$ and $y_{i}(t)$ of system (3) are extinct.

Corollary 16. If there is a constant $\lambda_{1}>0$ such that

$$
\begin{aligned}
& \liminf _{t \rightarrow+\infty} \int_{t}^{t+\lambda_{1}} b\left(s,\left(\frac{A_{x}}{\mu_{x}+\alpha_{x}}\right)^{m},\left(\frac{A_{y}}{\mu_{y}+\alpha_{y}}\right)^{m}\right) d s \\
& >0,
\end{aligned}
$$

then the infective $y_{i}(t)$ of system (3) is permanent.

Corollary 17. If there exists a constant $\lambda_{1}>0$ such that $\bar{R}_{01}>$ 1 , where

$$
\bar{R}_{01}=\frac{\left(3 \sqrt[3]{\beta_{x} \beta_{y} \theta \underline{N}_{x} \underline{N}_{y}}\right)_{0}}{\left(\left(\mu_{x}+\alpha_{x}\right)+\left(\mu_{y}+\alpha_{y}\right)+\left(\mu_{y}+\theta\right)\right)^{0}} .
$$


Here,

$$
\begin{aligned}
& \left(3 \sqrt[3]{\beta_{x} \beta_{y} \theta \underline{N}_{x} \underline{N}_{y}}\right)_{0} \\
& \quad=\liminf _{t \rightarrow+\infty} \int_{t}^{t+\lambda_{1}} 3 \sqrt[3]{\beta_{x}(t) \beta_{y}(t) \theta(t) \underline{N}_{x}(t) \underline{N}_{y}(t)} d t \\
& \quad\left(\left(\mu_{x}+\alpha_{x}\right)+\left(\mu_{y}+\alpha_{y}\right)+\left(\mu_{y}+\theta\right)\right)^{0} \\
& =\limsup _{t \rightarrow+\infty} \int_{t}^{t+\lambda_{1}}\left(\mu_{x}(t)+\alpha_{x}(t)\right)+\left(\mu_{y}(t)+\alpha_{y}(t)\right) \\
& +\left(\mu_{y}(t)+\theta(t)\right) d t
\end{aligned}
$$

$N_{x}(t)$ is some fixed solution of (8) with initial value $\underline{N}_{x}(0)>$ 0 and $\underline{N}_{y}(t)$ is some fixed solution of (10) with initial value $\underline{N}_{y}(0)>0$. Then the infective $y_{i}(t)$ of system (3) is permanent.

Corollary 18. Suppose system (3) is w-periodic; then the infective $y_{i}(t)$ of system (3) is permanent provided that

$$
\bar{R}_{02}=\frac{\overline{\left(3 \sqrt[3]{\beta_{x} \beta_{y} \theta \underline{N}_{x} \underline{N}_{y}}\right)}}{\overline{\left(\left(\mu_{x}+\alpha_{x}\right)+\left(\mu_{y}+\alpha_{y}\right)+\left(\mu_{y}+\theta\right)\right)}}>1 .
$$

Here, $\underline{N}_{x}(t)$ is the globally uniformly attractive nonnegative $\omega$-periodic solution of (8) and $\underline{N}_{y}(t)$ is the globally uniformly attractive nonnegative $\omega$-periodic solution of (10).

Corollary 19. Suppose system (3) is almost periodic; then the infective $y_{i}(t)$ of system (3) is permanent provided that

$$
\bar{R}_{03}=\frac{m\left(3 \sqrt[3]{\beta_{x} \beta_{y} \theta \underline{N}_{x} \underline{N}_{y}}\right)}{m\left(\left(\mu_{x}+\alpha_{x}\right)+\left(\mu_{y}+\alpha_{y}\right)+\left(\mu_{y}+\theta\right)\right)}>1 .
$$

Here, $\underline{N}_{x}(t)$ is the globally uniformly attractive nonnegative almost periodic solution of (8) and $\underline{N}_{y}(t)$ is the globally uniformly attractive nonnegative almost periodic solution of (10).

Corollary 20. Suppose that $\mu_{x}(t)>0, \mu_{y}(t)>0$ for all $t \geq 0$ and $0<\lim \inf _{t \rightarrow \infty}\left(A_{x}(t) /\left(\mu_{x}(t)+\alpha_{x}(t)\right)\right) \leq$ $\lim \sup _{t \rightarrow \infty}\left(A_{x}(t) / \mu_{x}(t)\right)<\infty, 0<\liminf _{t \rightarrow \infty}\left(A_{y}(t) /\right.$ $\left.\left(\mu_{y}(t)+\alpha_{y}(t)\right)\right) \leq \lim \sup _{t \rightarrow \infty}\left(A_{y}(t) / \mu_{y}(t)\right)<\infty$. If there exist positive constants $\lambda>0, p>0, q>0$, and $T_{1}>0$ such that

$$
\begin{gathered}
p>\frac{(\theta)^{0}}{\left(\mu_{y}+\alpha_{y}\right)_{0}} \\
q>\frac{\left(\beta_{x} \bar{N}_{x}\right)^{0}}{\left(\mu_{x}+\alpha_{x}\right)_{0}} \\
p q>\frac{\left(\mu_{y}+\theta\right)^{0}}{\left(\beta_{y} \bar{N}_{y}\right)_{0}}
\end{gathered}
$$

and $G_{1}(p, t)<0, G_{2}(p, q, t)<0$ for all $t \geq T_{1}$, then the two infectious populations $x_{i}(t)$ and $y_{i}(t)$ of system (3) are extinct. Here,

$$
\begin{gathered}
(\theta)^{0}=\limsup _{t \rightarrow+\infty} \int_{t}^{t+\lambda} \theta(s) d s, \\
\left(\mu_{y}+\theta\right)^{0}=\limsup _{t \rightarrow+\infty} \int_{t}^{t+\lambda}\left(\mu_{y}(s)+\theta(s)\right) d s, \\
\left(\beta_{x} \bar{N}_{x}\right)^{0}=\limsup _{t \rightarrow+\infty} \int_{t}^{t+\lambda} \beta_{x}(s) \bar{N}_{x}(s) d s, \\
\left(\beta_{y} \bar{N}_{y}\right)_{0}=\liminf _{t \rightarrow+\infty} \int_{t}^{t+\lambda} \beta_{y}(s) \bar{N}_{y}(s) d s, \\
\left(\mu_{y}+\alpha_{y}\right)_{0}=\liminf _{t \rightarrow+\infty} \int_{t}^{t+\lambda}\left(\mu_{y}(s)+\alpha_{y}(s)\right) d s, \\
\left(\mu_{x}+\alpha_{x}\right)_{0}=\liminf _{t \rightarrow+\infty} \int_{t}^{t+\lambda}\left(\mu_{x}(s)+\alpha_{x}(s)\right) d s .
\end{gathered}
$$

$\bar{N}_{x}(t)$ is some fixed solution of (9) with initial value $\bar{N}_{x}(0)>$ 0 and $\bar{N}_{y}(t)$ is some fixed solution of (11) with initial value $\bar{N}_{y}(0)>0$.

Corollary 21. Suppose system (3) is w-periodic; we suppose that $\mu_{x}(t)>0, \mu_{y}(t)>0$ for all $t \geq 0$ and $0<\lim \inf _{t \rightarrow \infty}\left(A_{x}(t) /\left(\mu_{x}(t)+\alpha_{x}(t)\right)\right) \leq \lim \sup _{t \rightarrow \infty}\left(A_{x}(t) /\right.$ $\left.\mu_{x}(t)\right)<\infty, 0<\lim _{\inf _{t \rightarrow \infty}}\left(A_{y}(t) /\left(\mu_{y}(t)+\alpha_{y}(t)\right)\right) \leq$ $\lim \sup _{t \rightarrow \infty}\left(A_{y}(t) / \mu_{y}(t)\right)<\infty$. Then if there exist positive constants $\lambda>0, p>0, q>0$, and $T_{1}>0$ such that

$$
\begin{gathered}
p>\frac{\overline{(\theta)}}{\overline{\left(\mu_{y}+\alpha_{y}\right)}}, \\
q>\frac{\overline{\left(\beta_{x} \bar{N}_{x}\right)}}{\overline{\left(\mu_{x}+\alpha_{x}\right)}}, \\
p q<\frac{\overline{\left(\mu_{y}+\theta\right)}}{\overline{\left(\beta_{y} \bar{N}_{y}\right)}}
\end{gathered}
$$

and $G_{1}(p, t)<0, G_{2}(p, q, t)<0$ for all $t \geq T_{1}$, then the two infectious populations $x_{i}(t)$ and $y_{i}(t)$ of system (3) are extinct. Here, $\bar{N}_{x}(t)$ is the globally uniformly attractive nonnegative $\omega$-periodic solution of (9) and $\bar{N}_{y}(t)$ is the globally uniformly attractive nonnegative $\omega$-periodic solution of (11).

Corollary 22. Suppose system (3) is almost periodic; also suppose that $\mu_{x}(t)>0, \mu_{y}(t)>0$ for all $t \geq 0$ and $0<\lim \inf _{t \rightarrow \infty}\left(A_{x}(t) /\left(\mu_{x}(t)+\alpha_{x}(t)\right)\right) \leq \lim \sup _{t \rightarrow \infty}\left(A_{x}(t) /\right.$ $\left.\mu_{x}(t)\right)<\infty, 0<\liminf _{t \rightarrow \infty}\left(A_{y}(t) /\left(\mu_{y}(t)+\alpha_{y}(t)\right)\right) \leq$ $\lim \sup _{t \rightarrow \infty}\left(A_{y}(t) / \mu_{y}(t)\right)<\infty$. Then if there exist positive 
constants $\lambda>0, p>0, q>0$, and $T_{1}>0$ such that

$$
\begin{gathered}
p>\frac{m(\theta)}{m\left(\mu_{y}+\alpha_{y}\right)}, \\
q>\frac{m\left(\beta_{x} \bar{N}_{x}\right)}{m\left(\mu_{x}+\alpha_{x}\right)}, \\
p q<\frac{m\left(\mu_{y}+\theta\right)}{m\left(\beta_{y} \bar{N}_{y}\right)}
\end{gathered}
$$

and $G_{1}(p, t)<0, G_{2}(p, q, t)<0$ for all $t \geq T_{1}$, then the two infectious populations $x_{i}(t)$ and $y_{i}(t)$ of system (3) are extinct. Here $\bar{N}_{x}(t)$ is the globally uniformly attractive nonnegative almost periodic solution of (9) and $\bar{N}_{y}(t)$ is the globally uniformly attractive nonnegative almost periodic solution of (11).

\section{Numerical Simulation}

Numerical verification of the results is necessary for completeness of the analytical study. In this section, we will present some numerical simulations to substantiate and discuss our analytical findings of system (3) by the means of the software Matlab.

In order to testify the validity of our results, in system (3), fix $A_{x}(t)=0.5+0.1 \sin (t), A_{y}(t)=0.6+0.1 \sin (t), \mu_{x}(t)=$ $0.2+0.18 \sin (t), \mu_{y}(t)=0.3+0.28 \sin (t), \alpha_{x}(t)=$ $0.4+0.3 \sin (t), \alpha_{y}(t)=0.5+0.2 \sin (t), \theta(t)=0.28+$ $0.2 \sin (t), \beta_{x}(t)=0.2+0.2 \sin (t), \beta_{y}(t)=0.1+0.1 \sin (t)$. It is easy to verify that (i) and (H2) hold. From (8)-(11), we have

$$
\begin{aligned}
& \lim _{t \rightarrow \infty} \underline{N}_{x}(t)=\frac{0.5+0.1 \sin (t)}{0.6+0.48 \sin (t)}, \\
& \lim _{t \rightarrow \infty} \bar{N}_{x}(t)=\frac{0.5+0.1 \sin (t)}{0.2+0.18 \sin (t)}, \\
& \lim _{t \rightarrow \infty} \underline{N}_{y}(t)=\frac{0.6+0.1 \sin (t)}{0.8+0.48 \sin (t)}, \\
& \lim _{t \rightarrow \infty} \bar{N}_{y}(t)=\frac{0.6+0.1 \sin (t)}{0.3+0.28 \sin (t)} .
\end{aligned}
$$

Then system (3) becomes periodic with period $2 \pi$. We choose $\lambda=2 \pi, p=3$, and $q=1$; then we have

$$
\begin{aligned}
G_{1}(p, t)= & -0.1267+0.0667 \sin (t)<0, \\
G_{2}(p, q, t)= & \frac{(0.2+0.2 \sin (t))(0.5+0.1 \sin (t))}{0.2+0.18 \sin (t)} \\
& -\frac{67}{75}-\frac{7}{15} \sin (t) .
\end{aligned}
$$

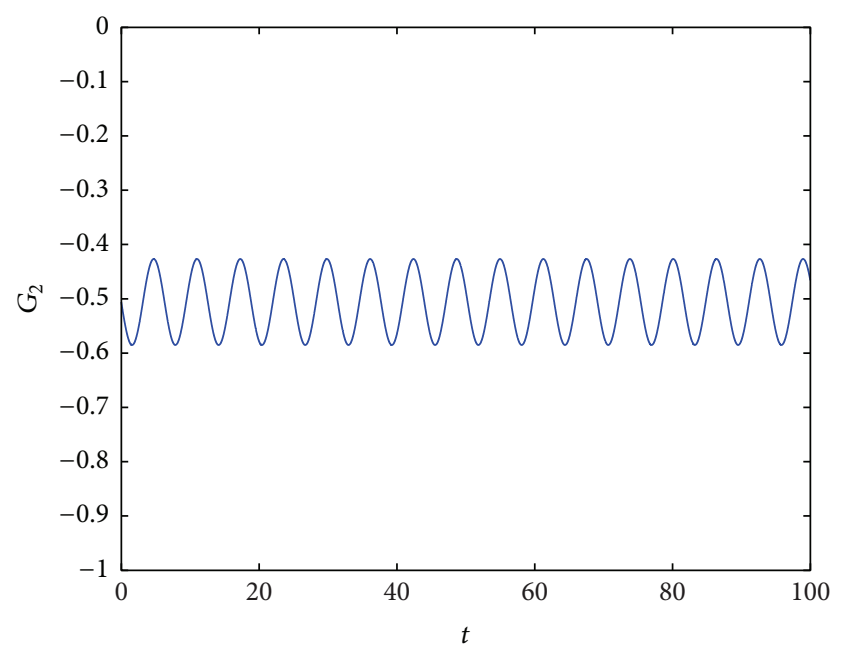

FIGURE 1: The figure shows that movement paths of $G_{2}(p, q, t)$ as functions of time $t$.

Obviously, it is easy to see that $G_{2}(p, q, t)<0$ for all $t>0$ from Figure 1. Furthermore, we can get that

$$
\begin{aligned}
& 3=p>\frac{\overline{(\theta)}}{\overline{\left(\mu_{y}+\alpha_{y}\right)}} \simeq 0.35, \\
& 1=q>\frac{\overline{\left(\beta_{x} \bar{N}_{x}\right)}}{\overline{\left(\mu_{x}+\alpha_{x}\right)}} \simeq 0.8279, \\
& 3=p q<\frac{\overline{\left(\mu_{y}+\theta\right)}}{\overline{\left(\beta_{y} \bar{N}_{y}\right)}} \simeq 3.2402 .
\end{aligned}
$$

Hence, from Corollary 21, we get that the disease will be extinct (see Figure 2). Increasing the infective rate to $\beta_{x}(t)=0.8+0.1 \sin (t), \beta_{y}(t)=0.5+0.1 \sin (t)$, from Corollary 18, we can get $\bar{R}_{02}=\overline{\left(3 \sqrt[3]{\beta_{x} \beta_{y} \theta \underline{N}_{x} \underline{N}_{y}}\right)} /$ $\overline{\left(\left(\mu_{x}+\alpha_{x}\right)+\left(\mu_{y}+\alpha_{y}\right)+\left(\mu_{y}+\theta\right)\right)}=4.1087>1$, which means the condition of Corollary 18 is satisfied. The conclusion of Corollary 18 is verified (see Figure 3).

\section{Parameters}

$A_{x}$ : Per capita birth rate of Rattus norvegicus

$\mu_{x}$ : Per capita natural death rate of Rattus norvegicus

$\alpha_{x}$ : Per capita disease induced death rate of Rattus norvegicus

$\beta_{x}$ : Per capita contact transmission rate from infected snails to susceptible Rattus norvegicus

$A_{y}:$ Per capita birth rate of snail host 


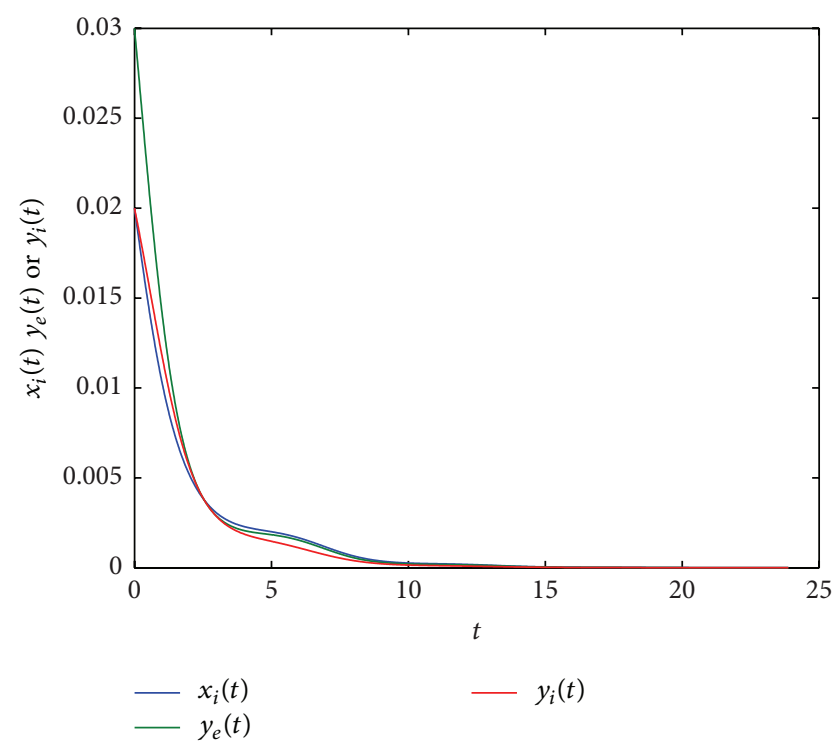

(a)

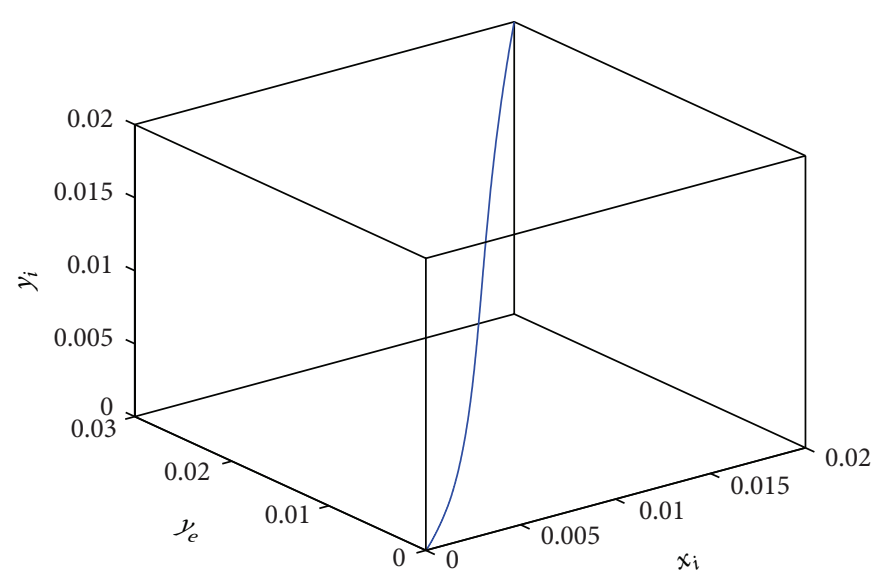

(b)

FIGURE 2: (a) shows movement paths of $x_{i}, y_{e}$, and $y_{i}$ as functions of time $t$. The graph of the trajectory in $\left(x_{i}, y_{e}, y_{i}\right)$-space is shown in (b). The disease is extinct.

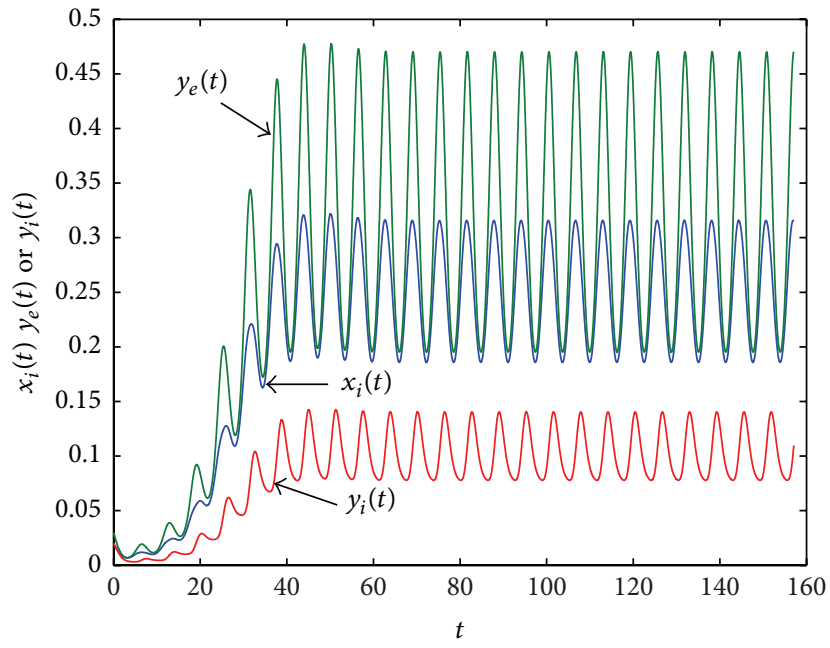

(a)

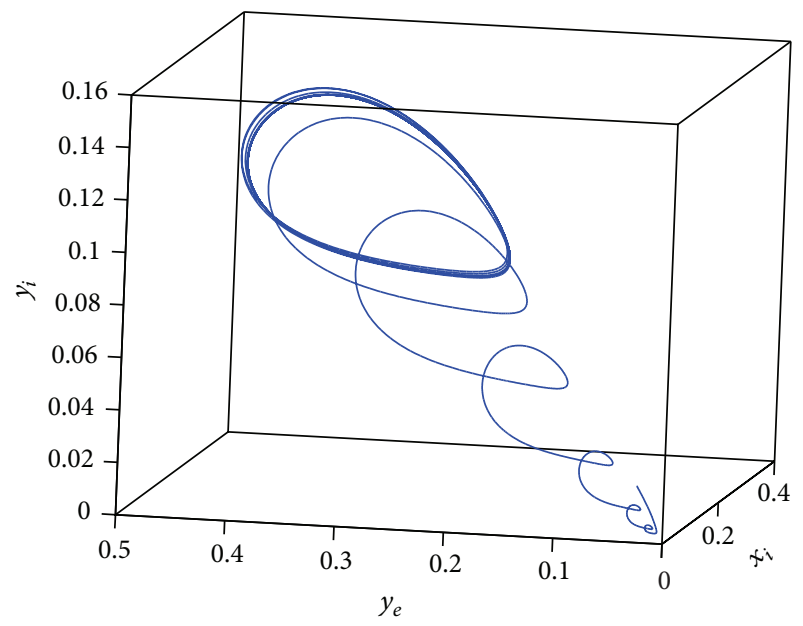

(b)

Figure 3: (a) shows movement paths of $x_{i}, y_{e}$, and $y_{i}$ as functions of time $t$. The graph of the trajectory in $\left(x_{i}, y_{e}, y_{i}\right)$-space is shown in (b). System (3) is permanent.

$\mu_{y}:$ Per capita natural death rate of snail host

$\alpha_{y}$ : Per capita disease induced death rate of snail host

$\beta_{y}$ : Per capita contact transmission rate from infected Rattus norvegicus to susceptible snails

$\theta$ : Per capita transition rate from infected and preshedding snails to shedding snails.

\section{Competing Interests}

The authors declare that they have no competing interests.

\section{Acknowledgments}

The research has been supported by the Natural Science Foundation of China (nos. 11261004 and 11561004), the Natural Science Foundation of Jiangxi Province (20151BAB201016), and the Supporting Development for Local Colleges and Universities Foundation of ChinaApplied Mathematics Innovative Team Building.

\section{References}

[1] WHO, Schistosomiasis, 2010, http://www.who.int/mediacentre/ factsheets/fs115/en/index.html. 
[2] Z. Feng, C.-C. Li, and F. A. Milner, "Schistosomiasis models with two migrating human groups," Mathematical and Computer Modelling, vol. 41, no. 11-12, pp. 1213-1230, 2005.

[3] G. Macdonald, "The dynamics of helminth infections, with special reference to schistosomes," Transactions of the Royal Society of Tropical Medicine and Hygiene, vol. 59, no. 5, pp. 489506, 1965.

[4] N. G. Hairston, "On the mathematical analysis of schistosome populations," Bulletin of the World Health Organization, vol. 33, no. 1, pp. 45-62, 1965.

[5] A. D. Barbour, "Modeling the transmission of schistosomiasis: an introductory view," The American Journal of Tropical Medicine and Hygiene, vol. 55, no. 5, pp. 135-143, 1996.

[6] E. T. Chiyaka and W. Garira, "Mathematical analysis of the transmission dynamics of schistosomiasis in the human-snail hosts," Journal of Biological Systems, vol. 17, no. 3, pp. 397-423, 2009.

[7] C. P. Bhunu, J. M. Tchuenche, W. Garira, G. Magombedze, and S. Mushayabasa, "Modeling the effects of schistosomiasis on the transmission dynamics of HIV/AIDS," Journal of Biological Systems, vol. 18, no. 2, pp. 277-297, 2010.

[8] Y. Yang and D. M. Xiao, "A mathematical model with delays for schistosomiasis japonicum transmission," Chinese Annals of Mathematics, Series B, vol. 31, no. 4, pp. 433-446, 2010.

[9] L. X. Qi and J. A. Cui, "Qualitative analysis for Barbour's schistosomiasis model with diffusion," Journal of Biomathematics, vol. 27, no. 1, pp. 54-64, 2012.

[10] L. X. Qi, J. A. Cui, Y. Gao, and H. P. Zhu, "Modeling the schistosomiasis on the islets in Nanjing," International Journal of Biomathematics, vol. 5, no. 4, Article ID 1250037, 17 pages, 2012.

[11] O. Diekmann, J. A. Heesterbeek, and J. A. Metz, "On the definition and the computation of the basic reproduction ratio $R_{\mathrm{o}}$ in models for infectious diseases in heterogeneous populations," Journal of Mathematical Biology, vol. 28, no. 4, pp. 365-382, 1990.

[12] O. Diekmann and J. A. P. Heesterbeek, Mathematical Epidemiology of Infectious Diseases: Model Building, Analysis and Interpretation, Wiley Series in Mathematical and Computational Biology, John Wiley \& Sons, Chichester, UK, 2000.

[13] Y. Chen, F. Chen, and Z. Li, "Dynamic behaviors of a general discrete nonautonomous system of plankton allelopathy with delays," Discrete Dynamics in Nature and Society, vol. 2008, Article ID 310425, 22 pages, 2008.

[14] G. P. Samanta, "Permanence and extinction for a nonautonomous avian-human influenza epidemic model with distributed time delay," Mathematical and Computer Modelling, vol. 52, no. 9-10, pp. 1794-1811, 2010.

[15] J. Hou, Z. D. Teng, and S. J. Gao, "Permanence and global stability for nonautonomous N-species Lotka-Valterra competitive system with impulses," Nonlinear Analysis: Real World Applications, vol. 11, no. 3, pp. 1882-1896, 2010.

[16] Y. Liu, H. Zhao, and J. Yan, "Existence of positive periodic solutions for $n$-dimensional nonautonomous system," Discrete Dynamics in Nature and Society, vol. 2014, Article ID 268418, 7 pages, 2014.

[17] L. Zhang and Z. Teng, "N-species non-autonomous LotkaVolterra competitive systems with delays and impulsive perturbations," Nonlinear Analysis: Real World Applications, vol. 12, no. 6, pp. 3152-3169, 2011.
[18] X. M. Feng and F. Q. Zhang, "The permanence in a single species nonautonomous system with delays and feedback control," Discrete Dynamics in Nature and Society, vol. 2010, Article ID 195983, 11 pages, 2010.

[19] G. P. Samanta, "Analysis of a delay nonautonomous predatorprey system with disease in the prey," Nonlinear Analysis: Modelling and Control, vol. 15, no. 1, pp. 97-108, 2010.

[20] S. H. Chen, T. X. Wang, and J. H. Zhang, "Positive periodic solution for non-autonomous competition Lotka-Volterra patch system with time delay," Nonlinear Analysis: Real World Applications, vol. 5, no. 3, pp. 409-419, 2004.

[21] J. Liu and T. Zhang, "Analysis of a nonautonomous epidemic model with density dependent birth rate," Applied Mathematical Modelling. Simulation and Computation for Engineering and Environmental Systems, vol. 34, no. 4, pp. 866-877, 2010.

[22] Y. Nakata and T. Kuniya, "Global dynamics of a class of SEIRS epidemic models in a periodic environment," Journal of Mathematical Analysis and Applications, vol. 363, no. 1, pp. 230237, 2010.

[23] T. Kuniya and Y. Nakata, "Permanence and extinction for a nonautonomous SEIRS epidemic model," Applied Mathematics and Computation, vol. 218, no. 18, pp. 9321-9331, 2012.

[24] T. Zhang and Z. Teng, "On a nonautonomous SEIRS model in epidemiology," Bulletin of Mathematical Biology, vol. 69, no. 8, pp. 2537-2559, 2007. 


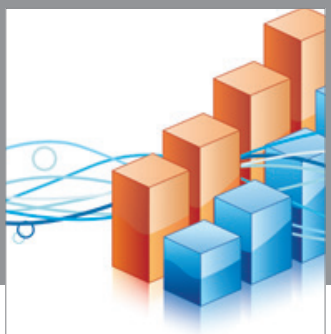

Advances in

Operations Research

vatem alat4

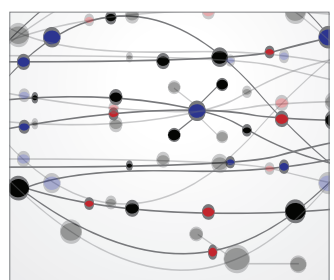

\section{The Scientific} World Journal
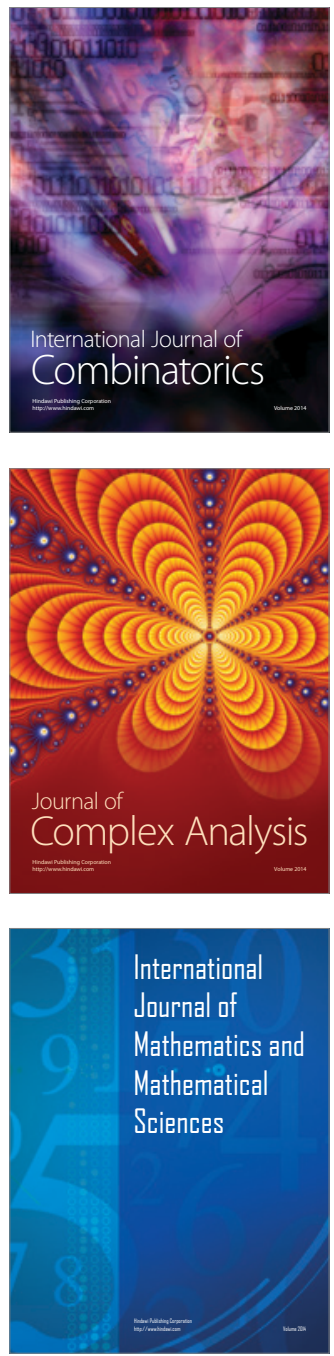
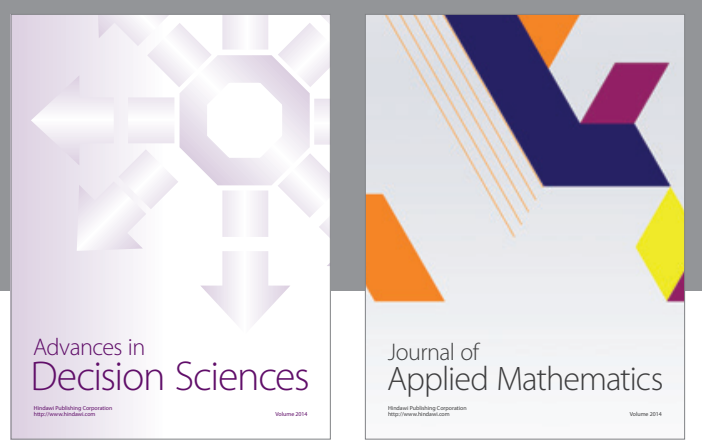

Algebra

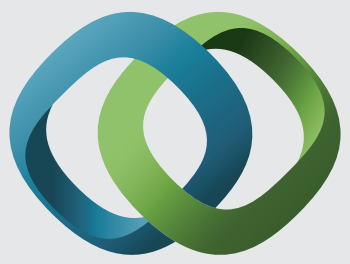

\section{Hindawi}

Submit your manuscripts at

http://www.hindawi.com
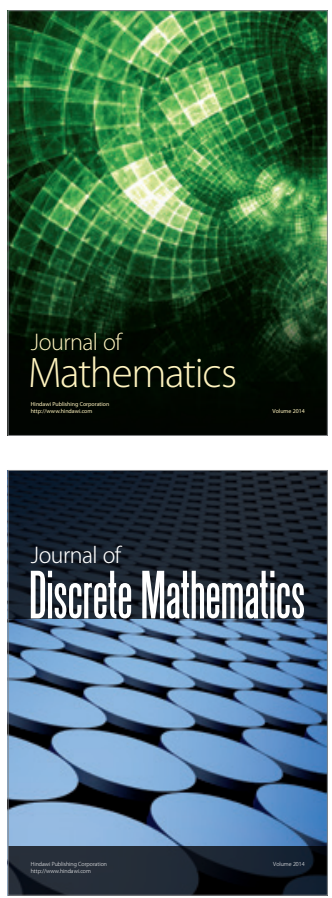

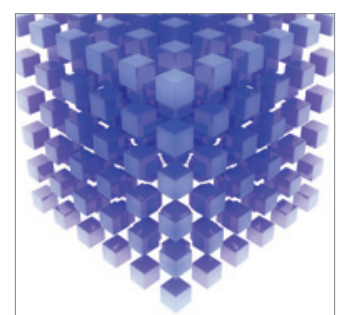

Mathematical Problems in Engineering
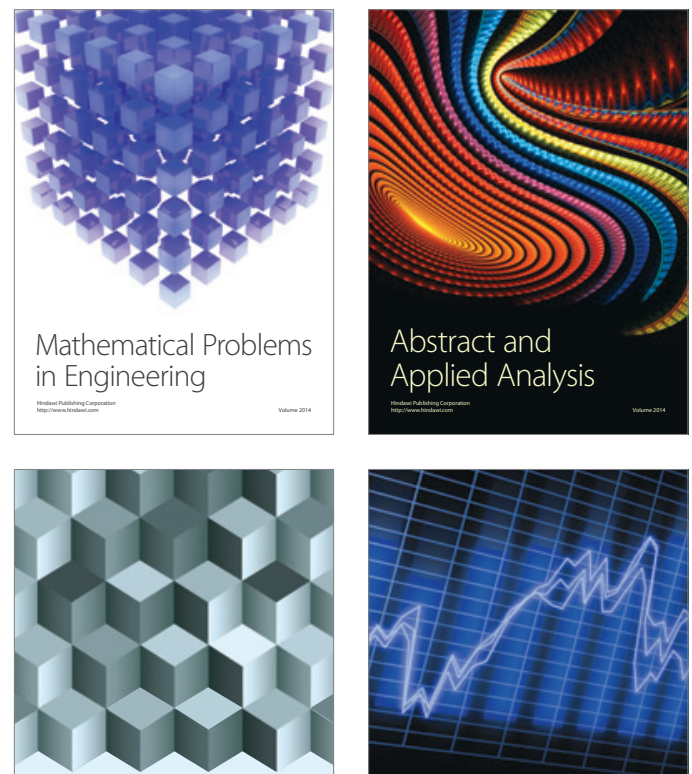

Journal of

Function Spaces

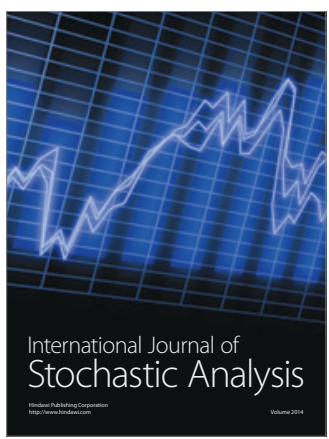

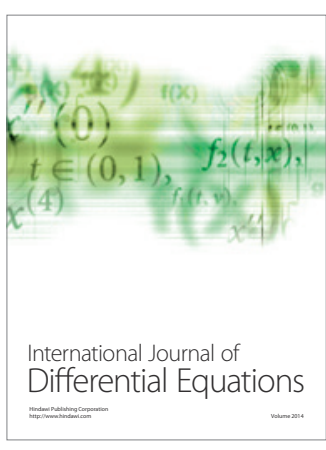
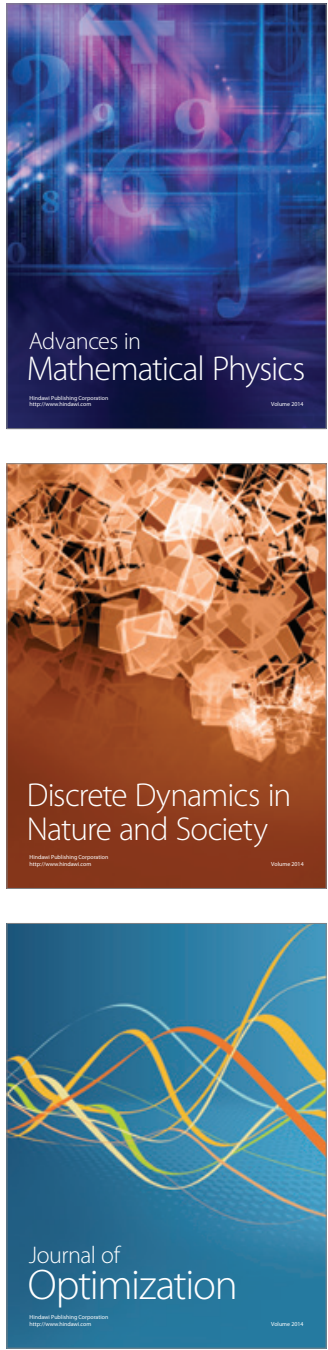